\title{
Melittin Constrains the Expression of Identified Key Genes Associated with Bladder Cancer
}

\author{
Zidan Jin $\mathbb{D}^{1,2}$ Jie Yao, ${ }^{3}$ Nianlin Xie $\mathbb{D}^{4},{ }^{4}$ Libo Cai, ${ }^{5}$ Shuai Qi, ${ }^{6}$ Zhan Zhang $\mathbb{D}^{1}$ and Bai Li ${ }^{1}$ \\ ${ }^{1}$ Department of Rehabilitation Medicine, Changhai Hospital, Second Military Medical University, Shanghai 200433, China \\ ${ }^{2}$ Department of Urological Surgery, The 161th Hospital of PLA, Wuhan, Hubei Province 430012, China \\ ${ }^{3}$ Department of Urological Surgery, Zhongnan Hospital of Wuhan University, Wuhan, Hubei Province 430071, China \\ ${ }^{4}$ Department of Thoracic Surgery, Tangdu Hospital, Fourth Military Medical University, Xi'an, Shanxi Province 710038, China \\ ${ }^{5}$ Department of Accident and Emergency, Heihe No.1 People's Hospital, Heihe 164300, China \\ ${ }^{6}$ Department of Pharmacy, The 161th Hospital of PLA, Wuhan, Hubei Province 430012, China
}

Correspondence should be addressed to Zhan Zhang; zzerato@163.com and Bai Li; libaikf@smmu.edu.cn

Received 22 January 2018; Revised 2 March 2018; Accepted 2 April 2018; Published 3 May 2018

Academic Editor: Senthamil R. Selvan

Copyright ( 2018 Zidan Jin et al. This is an open access article distributed under the Creative Commons Attribution License, which permits unrestricted use, distribution, and reproduction in any medium, provided the original work is properly cited.

\begin{abstract}
This work is aimed at investigating the effect of melittin on identified key genes in bladder cancer (BC) and further providing a theoretical basis for BC treatment. GSE35014 downloaded from the Gene Expression Omnibus (GEO) database was used to screen differentially expressed genes (DEGs) in BC cells and control. Results showed that a total of 389 upregulated and 169 downregulated genes were identified. Subsequently, GO analysis, KEGG pathway enrichment analysis, and PPI network analysis were employed to disclose the crucial genes and signaling pathways involved in BC. Fifteen module-related DEGs and their associated signaling pathways were obtained according to the PPI network and modular analyses. Based on the analysis of articles retrieved in the PubMed database, we found that melittin could induce apoptosis and constrain the progression of tumor cells as a result of regulating critical cancer-related signaling pathways, such as PI3K-Akt and TNF signaling pathways. Furthermore, PI3K-Akt and TNF signaling pathways were also found to be associated with module-related DEGs according to biological analyses. At last, qRT-PCR analysis demonstrated that melittin could constrain the expression of module-related DEGs (LPAR1, COL5A1, COL6A2, CXCL1, CXCL2, and CXCL3) associated with PI3K-Akt and TNF signaling pathways in BC cells. Functional assays revealed that melittin could constrain the proliferative and migrated abilities of $\mathrm{BC}$ cells. Conjointly, these findings provide a theoretical basis for these six genes as drug-sensitive markers of melittin in BC treatment.
\end{abstract}

\section{Introduction}

Commonly accepted, bladder cancer (BC) pertains to a malignant tumor with top incidence in urological diseases [1]. Overall, bladder tumors are commonly in the presence of noninvasive urothelial carcinoma and muscleinvasive disease [2]. The invasive subtype of $\mathrm{BC}$ is closely associated with metastatic spread and poor prognosis of patients [3]. Despite great advances made in the diagnosis and treatment of $\mathrm{BC}$, therapeutic options are limited and prognosis remains unfavorable $[4,5]$. Therefore, elucidating the underlying molecular mechanism of development and progression is conductive to and even decisive on BC treatment.
The application of Chinese traditional medicine in tumor treatment has gained much attention in recent years [6-8]. Melittin with twenty-six amino acids is the essential component of honeybee venom [9]. Prior research generally confirms that honeybee venom is accepted as Chinese medicine and applied for treatment of various diseases, exemplified by arthritis, rheumatism, back pain, cancer, and skin disease [10]. Intriguingly, discussions regarding melittin associated with diverse cancers have dominated research in recent years. In our previous work, we retrieved and analyzed articles from 2010 to now in the PubMed database with the search keyword of melittin and cancer. We found that melittin could induce apoptosis and constrain the progression of tumor cells as a result of regulating critical cancer-related signaling 
pathways. For example, Jo et al. unveiled that melittin could have an anticancer effect as a result of inducing apoptotic cell death in ovarian cancer through enhancement of death receptors and suppression of the JAK2/STAT3 signaling pathway [11]. A similar anticancer effect in prostate cancer cells was evidenced and materialized through activation of the caspase pathway via inactivation of NF- $\kappa \mathrm{B}$ [12]. It has also been reported that melittin could suppress PMA-induced tumor cell invasion by inhibiting NF- $\kappa \mathrm{B}$ - and AP-1-dependent MMP-9 expression [13], probably through JNK/p38- and NF- $\kappa \mathrm{B}-$ dependent mechanisms [14]. Jeong et al. outlined that melittin suppresses TNF- $\alpha$-induced HASMC migration through the selective inhibition of MMP-9 expression [15]. Shin et al. illustrated that melittin could hold down HIF-1 $\alpha$ /VEGF expression through inhibition of the ERK and mTOR/p70S6K pathway in human cervical carcinoma cells [16]. Jeong et al. probed that melittin could constrain EGF-induced MMP-9 expression via blocking the NF- $\kappa \mathrm{B}$ and PI3K/Akt/mTOR signaling pathway and repress EGF-induced FAK phosphorylation through inhibiting the mTOR/p/0S6K/4E-BP1 signaling pathway in breast cancer cells [17]. Kong et al. proffered the conclusion of melittin exerting a positive role in gastric cancer cell apoptosis via activation of the mitochondrial pathway [18]. Furthermore, the antitumor activity of melittin was reported to be associated with antiangiogenic actions via inhibiting VEGFR-2 and inflammatory mediators involved in the MAPK signaling pathway [19] or to suppress hepatic epithelial-to-mesenchymal transition via blocking of TGF $\beta / S$ mad and MAPK-JNK signaling pathways [20]. As recently reported, it was demonstrated that melittin could constrain tumor angiogenesis modulated by endothelial progenitor cells involved in the SDF- $1 \alpha /$ CXCR 4 signaling pathway in a UMR-106 osteosarcoma xenograft mouse model [21]. However, little was known about the association between melittin and $\mathrm{BC}$, which promoted us to conduct this study.

Popularly accepted, the Gene Expression Omnibus (GEO) accessible at www.ncbi.nlm.gov/geo is a public repository that archives and freely distributes high-throughput gene expression data submitted by the scientific community. Huge volumes of data including individual gene expression, multifarious organisms, and a wide range of biological issues could be effectively explored, queried, and visualized by means of user-friendly web-based tools. In recent years, microarrays contingent on high-throughput platforms have emerged as a promising and efficient tool for screening significant genetic or epigenetic alternations in carcinogenesis and identifying promising biomarkers for diagnosis and prognosis of cancers [22]. Plentiful gene expression profiling microarrays have been employed to identify multifarious differentially expressed genes (DEGs) in various cancers, and a number of BC-related DEGs have therefore been documented [23-25]. Nonetheless, various additional critical genes and pathways associated with $\mathrm{BC}$ remain yet to be explored completely.

In the present study, we aimed to disclose the crucial genes associated with $\mathrm{BC}$ and investigate the effect of melittin on the identified crucial genes in BC. On the one hand, the transcription profile of GSE35014 was downloaded from the GEO database (https://www.ncbi.nlm.nih.gov/geo/query/ acc.cgi?acc=GSE35014) and data processing was performed using $\mathrm{R}$ software (version 3.3.0, available online: https:// www.r-project.org/) and bioconductor packages (available online: http://www.bioconductor.org/), together with the online website Database for Annotation, Visualization, and Integrated Discovery (DAVID; version 6.8). Differentially expressed genes (DEGs) were screened in BC cells and control. Gene ontology (GO) analysis, Kyoto Encyclopedia of Genes and Genomes (KEGG) pathway enrichment analysis, and protein-protein interaction (PPI) network analysis were employed to evaluate the crucial genes and signaling pathways involved in BC. On the other hand, we collected melittin-related articles involved in cancers from the PubMed database and analyzed the relationship between melittin and various cancers that have been reported. After that, we combined the KEGG, PPI network, and module analyses intersecting with the literature analysis to obtain our target signaling pathway and crucial DEGs. The data analysis pipeline for key genes in BC is displayed in Figure 1. Finally, we investigated the effect of melittin on the critical DEG expression in $\mathrm{BC}$ cells and its functional role in proliferative and migrated abilities of $\mathrm{BC}$ separately by qRT-PCR and functional assays. By this token, these critical DEGs and signaling pathways identified in this work would convey valuable information about the mechanism of $\mathrm{BC}$ carcinogenesis and expose the discovery of key genes that may act as future targets of anticancer therapy.

\section{Materials and Methods}

2.1. Dataset Collection and Identification of DEGs in BC. Microarray technology becomes widely accepted as a highthroughput tool for measuring gene expression [26]. As previously reported, data from DNA microarray analysis plays a reliable and useful role in identifying novel targets for clinical diagnostic and therapeutic approaches $[27,28]$. The selection criteria of datasets in our work were described as follows: (1) associated with BC cells, (2) focus on the invasion and metastasis of BC, (3) sample size no less than 4, and (4) based on the GPL570 platform. Extracted from the GEO (https://www .ncbi.nlm.nih.gov/geo/) database, one microarray expression profile termed GSE35014 was therefore employed to identify DEGs associated with BC (https://www.ncbi.nlm.nih.gov/ geo/query/acc.cgi?acc $=$ GSE35014). The microarray data of GSE35014 was contingent on GPL570 platforms (Affymetrix Human Genome U133 Plus 2.0 Array, Affymetrix Inc., Santa Clara, CA, USA). It has been reported that UM-UC-3 bladder carcinoma cells have lost the endogenous expression of Rho GDP dissociation inhibitor 2 (RhoGDI2), as occurs commonly in the progression of $\mathrm{BC}$ [29]. In this query, two paired biological replicates of GFP (control) and GFP-RhoGDI2 (experimental) UM-UC-3 cells were constructed at different times, isolated during log phase growth, and subjected to gene expression profiling by means of the Affymetrix HG-U133 Plus 2.0 oligonucleotide microarray platform.

$\mathrm{R}$ software (version 3.3.0; https://www.r-project.org/) and bioconductor packages (available online: http://www .bioconductor.org/) were applied for data mining and 


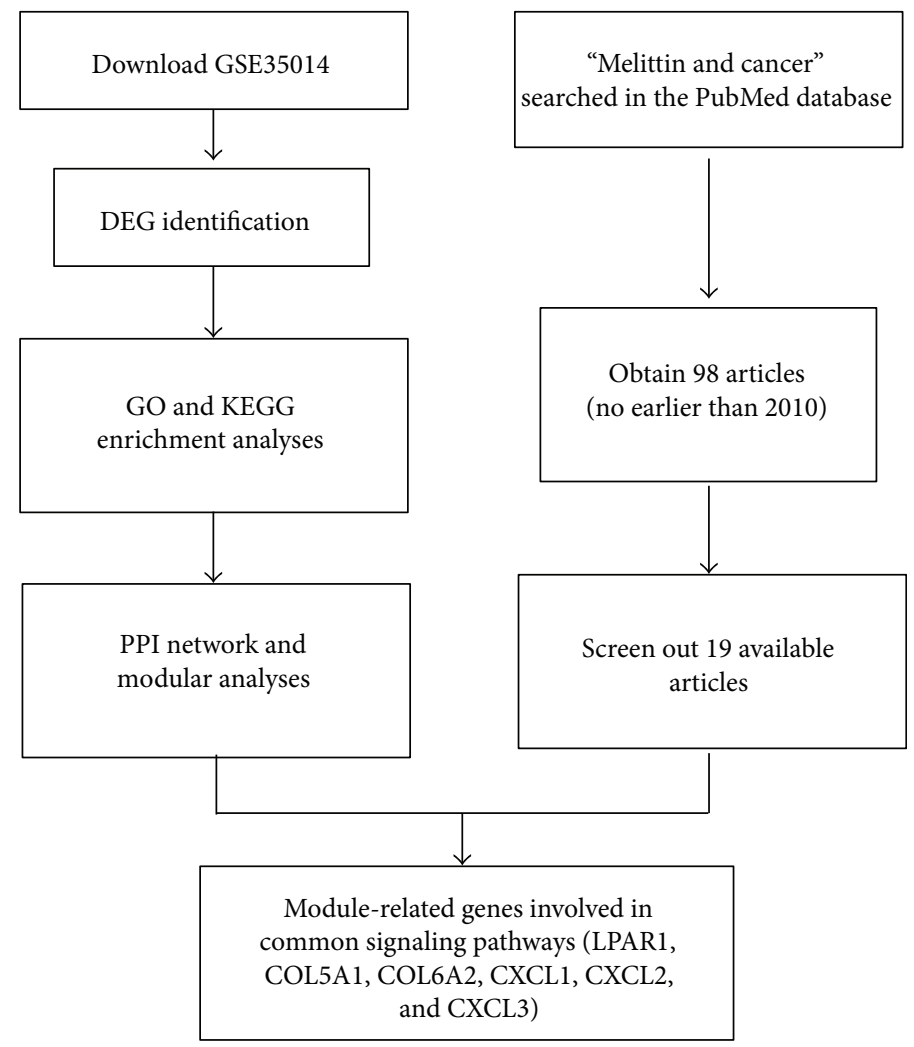

FIGURE 1: Data analysis pipeline for key genes in BC. DEGs: differentially expressed genes; GO: gene ontology; KEGG: Kyoto Encyclopedia of Genes and Genomes; PPI: protein-protein interaction; BC: bladder cancer.

statistical analyses. Concretely, the original annotation file and CEL files of the Affymetrix platform were firstly downloaded. Limma package [30] was applied to identify DEGs between experimental and control groups with the cut-off criterion of $P<0.05$ and absolute $\log 2 \mathrm{FC}>1$.

2.2. GO and KEGG Enrichment Analyses in BC. With regard to the investigation of potential biological functions of DEGs in BC, GO [31] functional and KEGG [32] pathway analyses were conjointly carried out. The DAVID online tool available at https://david.ncifcrf.gov/ was applied for these analyses. Significantly enriched GO terms and KEGG pathways were screened out as a criteria of $P<0.05$, finally visualized using $\mathrm{R}$ package ggplot2 [33].

2.3. PPI Network Construction. Search Tool for the Retrieval of Interacting Genes (STRING) accessible at http://string$\mathrm{db}$. org/ is an online database which includes experimental data and computational prediction methods as well as public text collections, involved in beyond 1100 completely sequenced organisms [34]. The protein interactions in the STRING database were presented as a combined score. The STRING database system was used to construct the functional protein association networks. Interacting pairs with a combined score more than 0.8 were considered to be significant and employed to construct networks. Cytoscape (http://www.cytoscape.org/) [35] was disseminated to visualize these networks.
2.4. MCODE Analysis. Molecular Complex Detection (MCODE; https://omictools.com/molecular-complex-detection-tool) could be applied to evaluate densely connected regions in large PPI networks possibly involved in molecular complexes [36]. MCODE was disseminated to mine the core protein complex in the PPI network constructed in this work.

2.5. Cell Culture and Treatment. The human $\mathrm{BC}$ cell lines T24 and 5637 were purchased from the American Type Culture Collection (ATCC; Manassas, VA). Cells were cultured in Dulbecco's modified Eagle's medium (DMEM; Gibco BRL, Grand Island, NY, USA) supplemented with $10 \%$ fetal bovine serum (FBS; Gibco BRL, Grand Island, NY, USA) and $100 \mathrm{U} / \mathrm{ml}$ of penicillin/streptomycin. The cultured cells were maintained at $37^{\circ} \mathrm{C}$ in a humidified atmosphere with $5 \%(v / v) \mathrm{CO}_{2}$. Cells were plated in 24-well plates $\left(5 \times 10^{4}\right.$ cells/well), and subconfluent cells were treated with melittin at a concentration of 0 and $4 \mu \mathrm{g} / \mathrm{ml}$ for $24 \mathrm{~h}$, respectively. Melittin used in this work was purchased from SigmaAldrich (St. Louis, MO, USA).

2.6. Real-Time Quantitative PCR Validation. Total RNA was extracted from treated cells using the TRIzol reagent (Life Technologies, California, USA) according to the manufacturer's instructions. $1 \mu \mathrm{g}$ of the total RNA was withdrawn and used as the template for cDNA synthesis using a PrimeScript RT Reagent Kit with cDNA Eraser (Takara Biotech, Dalian, China). Quantitative real-time polymerase chain reaction (qRT-PCR) was performed by using SYBR Premix 
TABLE 1: The primers for qRT-PCR analysis were applied in this work.

\begin{tabular}{lcc}
\hline Genes & Orientation & Sequences $\left(5^{\prime}-3^{\prime}\right)$ \\
\hline \multirow{2}{*}{ LPAR1 } & Forward & GGCTGCCATCTCTACTTCCATCC \\
& Reverse & ACAAACAGTGATTCCAAGTCCCA \\
COL5A1 & Forward & ATCTTCGGCTCTCTCAACTCTC \\
& Reverse & GTCCACATAGGAGAGCAGTTTC \\
COL6A2 2 Forward & Reverse & AGCCTACGGAGAGTGCTACAAG \\
& Forward & TGTCCATCGGTCCCGTTCTTGC \\
CXCL1 & Reverse & AACCGAAGTCATAGCCACA \\
& Forward & TCCTAAGCGATGCTCAAA \\
CXCL2 & Reverse & CAAACCGAAGTCATAGCC \\
& Forward 3 GAACAGCCACCAATAAGC \\
GAPDH & Reverse & CAAACCGAAGTCATAGCC \\
& Forward & ACCCTCGTAAGAAATAGTCA \\
\hline
\end{tabular}

Ex Taq (Takara Biotech, Japan) on an ABI 7900 system (Applied Biosystems, Foster City, CA, USA). The primers used in this work are presented in Table 1. The housekeeping gene glyceraldehyde 3-phosphate dehydrogenase (GAPDH) was used as an internal control. The quantification of gene expression was calculated using a relative quantification $\operatorname{method}\left(2^{-\Delta \Delta C t}\right)$.

2.7. Cell Proliferation Assays. After treatment with melittin (0 and $4 \mu \mathrm{g} / \mathrm{ml})$, cell proliferation was assessed by the MTS assay (Promega, Madison, US) according to the manufacturer's protocol. 5637 and T24 cells (2000 cells per well) in each group were plated in 96-well plates. Twenty microliters of the MTS reagent was added to each well containing $100 \mu \mathrm{l}$ of culture medium. The plate was incubated for $2 \mathrm{~h}$ at $37^{\circ} \mathrm{C}$ in a humidified atmosphere with $5 \%(v / v) \mathrm{CO}_{2}$. The plate was then read at $490 \mathrm{~nm}$ using a plate reader.

2.8. Cell Migration Assays. The scratch wound-healing assay was employed to examine cell migration. Uniform wounds were scraped in 5637 and T24 cells grown on plastic 6-well plates using a pipette tip before transfection. The initial gap length $(0 \mathrm{~h})$ and the residual gap length $(48 \mathrm{~h})$ after wounding were calculated from photomicrographs.

2.9. Statistical Analysis. Each experiment was repeated three times, and all values were presented as the means \pm standard deviation (SD). The $t$-test was employed to analyze the difference in two groups. GraphPad Prism software version 5.0 was applied to complete statistical analysis. A value of $P<0.05$ was considered statistically significant in this work.

\section{Results}

3.1. Identification of DEGs in BC. According to microarray analysis, a total of 558 DEGs were achieved to be differentially expressed with a fold change $>2$ between the BC cell group and control, as revealed in Figure 2. Among them, 389 upregulated and 169 downregulated genes were separately obtained as listed in Table 2. These DEGs were employed for follow-up analyses.

3.2. GO Enrichment Analysis. Commonly, GO functional enrichment analysis is involved in biological process, molecular function, and cellular component. In this study, GO functional enrichment analysis of DEGs was performed by means of an online biological tool DAVID with a threshold of $P<0.05$. Top thirty significant GO terms according to $P$ value are visualized and displayed in Figure 3, and corresponding related DEGs are summarized in Table 3 . It could be materialized that a sum of DEG-enriched terms play a critical role in the biological behavior of tumor cells. Most of the DEGs were significantly enriched in protein binding, plasma membrane, extracellular space, and signal transduction. Concretely, in terms of biological process, DEGs were principally enriched in signal transduction, cell adhesion, and positive regulation of cells, while they are concentrated in the plasma membrane, extracellular space, and cell surface with respect to cellular component. As for molecular function, DEGs focused on the relation with protein binding, actin binding, and heparin binding.

3.3. Signaling Pathway Enrichment Analysis. Pathway analyses were used to identify the significant pathways associated with the DEGs according to KEGG analysis. As mentioned in GO analysis, the online biological tool DAVID with a threshold of $P<0.05$ was also applied in this section. Top fifteen significant pathways were visualized by $R$ package ggplot2. As depicted in Figure 4 and Table 4, DEGs were essentially abundant in cancer-related pathways, such as pathways in cancer (ID: hsa05200), PI3K-Akt signal pathway (ID: hsa04151), cytokine-cytokine receptor interaction (ID: hsa04060), focal adhesion (ID: hsa04510), cell adhesion molecules (ID: hsa04514), transcriptional misregulation in cancer (hsa05202), Jak-STAT signal pathway (ID: hsa04630), and TNF signaling pathway (ID: hsa04668), which all play a critical and even decisive role in the progression and development of tumors. 


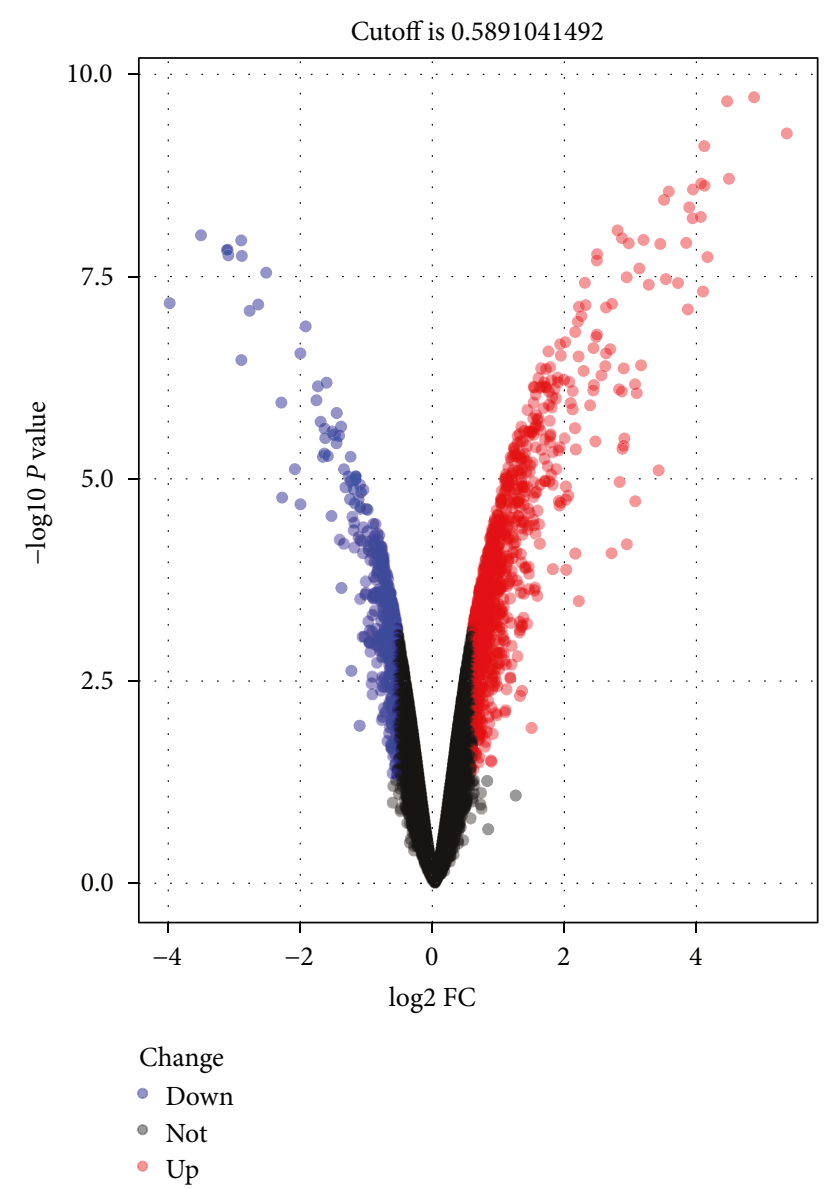

FIGURE 2: Volcano plot of detectable DEG profiles in BC cells. Red plots stand for upregulated genes, and blue ones represent downregulated genes with $P<0.05$ and absolute $\log _{2}$ FC $>1$. Black ones indicate those nonsignificant expressed genes. The abscissa means the value of fold change in circulating the gene expression between $\mathrm{BC}$ cells and control. The ordinate shows the $-\log _{10}$ of the adjusted $P$ value for each gene, suggesting the strength of the association. DEGs: differentially expressed genes; BC: bladder cancer; FC: fold change.

3.4. Identification of Key Candidate Genes and Signaling Pathways Based on PPI Network and Modular Analyses. With the purpose of systemically analyzing the functions of DEGs in BC, these DEGs were mapped to PPI data dependent on the STRING online database (available online: http://string-db.org) and Cytoscape software (version 3.4.0, available online: http://www.cytoscape.org/). Interacting pairs with a combined score more than 0.8 were considered to be significant and employed to construct networks. The PPI network with 309 interacting pairs was thus obtained (Figure 5(a)), which also was provided as Supplementary Material (available here) for zooming in and out as needed. Based on the PPI network, we subsequently analyzed the network clusters by MCODE, and twelve module networks were therefore generated as described in Table 5 . The module networks with a score no less than 4 were selected for the next analysis and are depicted in Figure 5(b). Fifteen modulerelated DEGs marked as bold in Table 4 were thus obtained, including CXCL2, CXCL1, BDKRB1, LPAR1, CXCL3, FYN,
COL6A2, COL18A1, COL13A1, COL5A1, TUBA1A, FDFT1, SQLE, LSS, and CDK9. Combined with Tables 4 and 5, it could be found that the signaling pathways associated with these module-related DEGs were described as follows: pathways in cancer (ID: hsa05200), PI3K-Akt signaling pathway (ID: hsa04151), focal adhesion (ID: hsa04510), transcriptional misregulation in cancer (ID: hsa05202), TNF signaling pathway (ID: hsa04668), Gap junction (ID: hsa04540), protein digestion and absorption (ID: hsa04974), and steroid biosynthesis (ID: hsa00100).

3.5. Melittin Constrained the Expression of the ModuleRelated DEGs Associated with PI3K-Akt and TNF Signaling Pathways in BC Cells. According to literature analysis, we found that the signaling pathways associated with melittin in cancers mainly include JAK2/STAT3, NF- $\kappa \mathrm{B}$, JNK/p38/ NF- $\kappa$ B, TNF- $\alpha$, mTOR/p70S6K or mTOR/p/0S6K/4E-BP1, $\mathrm{PI} 3 \mathrm{~K} / \mathrm{Akt} / \mathrm{mTOR}$, mitochondrial pathway, MAPK signaling pathway, TGF $\beta /$ Smad and MAPK-JNK signaling pathways, and SDF-1 $\alpha /$ CXCR4 signaling pathway so far [11-21]. JAK2/STAT3, TNF- $\alpha, \mathrm{mTOR} / \mathrm{p} 70 \mathrm{~S} 6 \mathrm{~K}$ or mTOR/ $\mathrm{p} / 0 \mathrm{~S} 6 \mathrm{~K} / 4 \mathrm{E}-\mathrm{BP} 1$, and PI3K/Akt/mTOR signaling pathways represented above were in accordance with the signaling pathway IDs of hsa04630, hsa04668, hsa04150, and hsa04151 as shown in Table 4. The module-related DEGs in the PI3K/Akt (namely, LPAR1, COL5A1, and COL6A2) and TNF signaling pathway (termly CXCL1, CXCL2, and CXCL3) were selected to investigate the effect of melittin on them in BC cells. As revealed in Figure 6, it was clear that the expressions of LPAR1, COL5A1, and COL6A2 associated with the PI3K/Akt signaling pathway were significantly decreased in cells treated with melittin compared to the control. So was the case in the expressions of CXCL1, CXCL2, and CXCL3 involved in the TNF signaling pathway. Collectively, our findings demonstrated that melittin could constrain the expression of the modulerelated DEGs associated with PI3K-Akt and TNF signaling pathways in BC cells.

3.6. Melittin Suppressed Cell Proliferation and Migration in $B C$ Cell Lines. As previously reported, melittin could have an inhibitory effect on cell motility and migration through PI3K/Akt and TNF signaling pathways in diseases $[15,17]$. We therefore investigated the effect of melittin on cell proliferation and migration in 5637 and T24 cell lines by MTS and scratch wound-healing assays. Results obtained showed that cell viabilities were significantly inhibited in the melittintreated group $(4 \mu \mathrm{g} / \mathrm{ml})$ in comparison with the control group $(0 \mu \mathrm{g} / \mathrm{ml})$ according to the MTS assay (Figures $7(\mathrm{a})$ and $7(\mathrm{~b}), P<0.05)$. Similar results were also obtained in the effect of melittin on cell migration as analyzed by the scratch wound-healing assay (Figures $7(\mathrm{c})$ and $7(\mathrm{~d}), P<0.05$ ). Taken together, melittin could induce a significant reduction in proliferation and migration of $\mathrm{BC}$ cells.

\section{Discussion}

As we know, melittin with twenty-six amino acids is the essential component of honeybee venom [9]. Current 


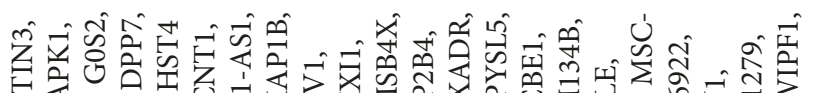

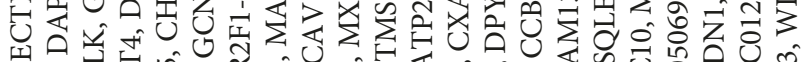

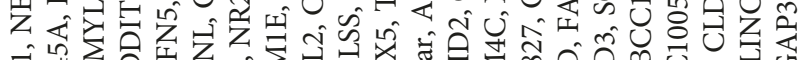

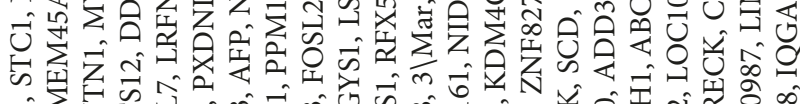

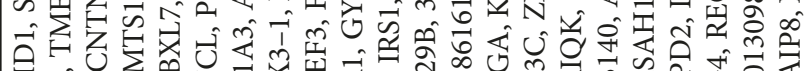
年

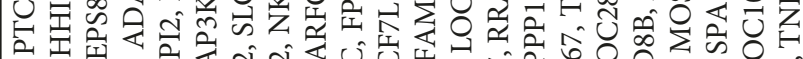
(1)

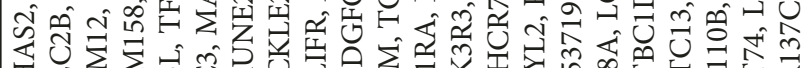

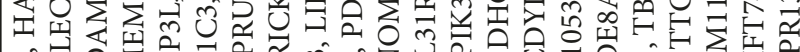

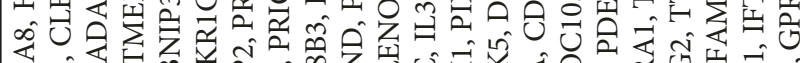

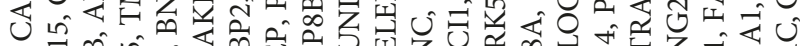

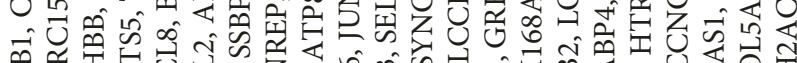

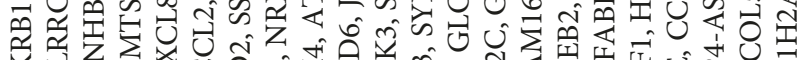

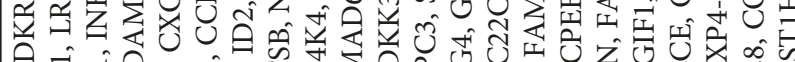

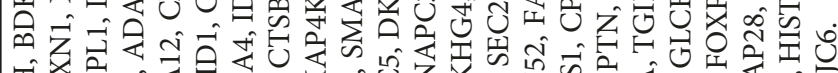

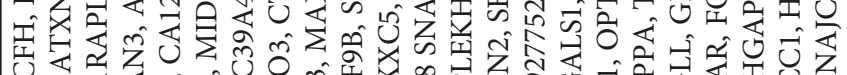

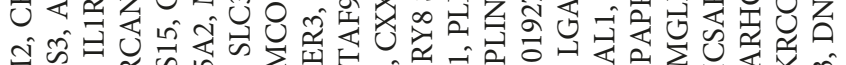

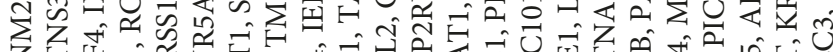

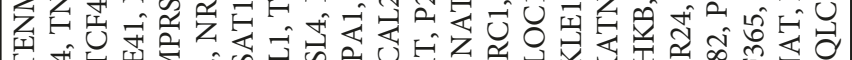

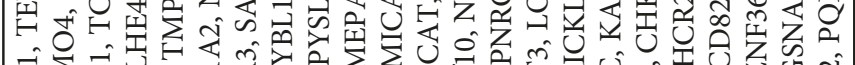

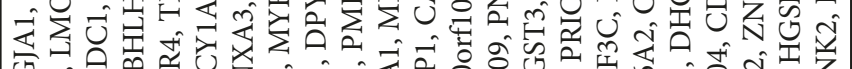
0

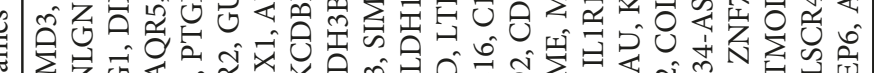

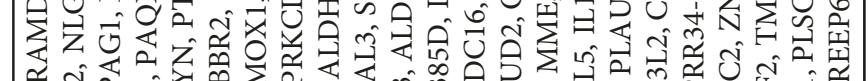

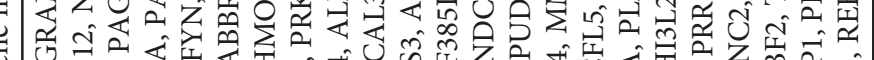

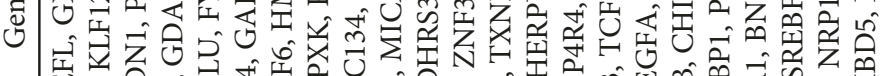

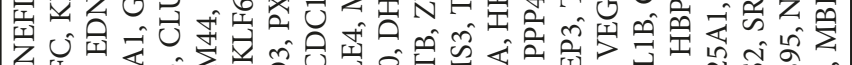

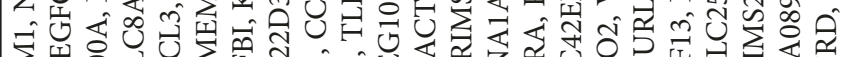

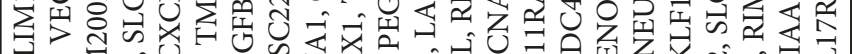
常 $=\sum_{1}$ की

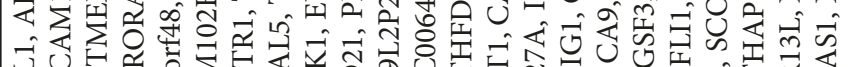
ज्ञ

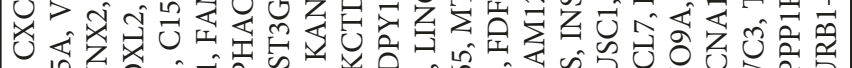
ी

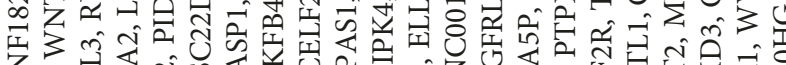

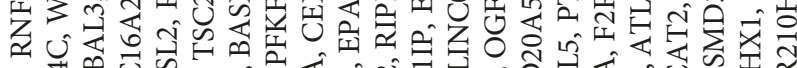

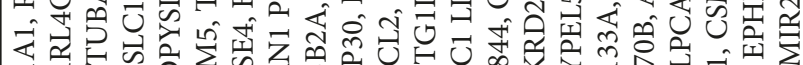

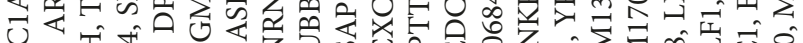
ง̂ี่

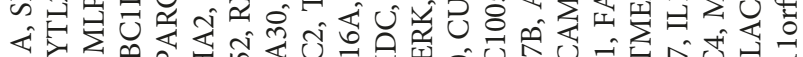

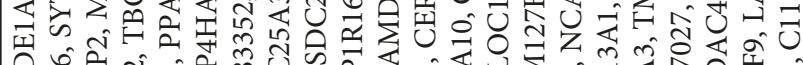

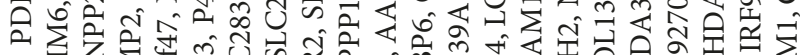

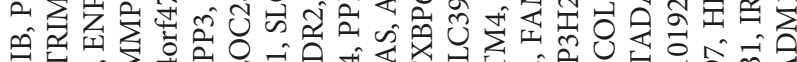

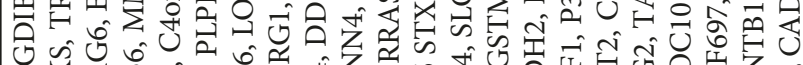
ज̂ञ

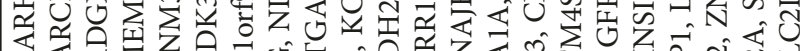

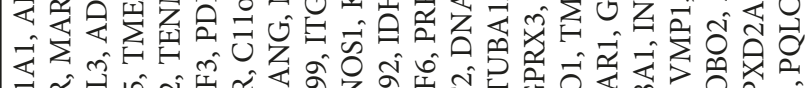

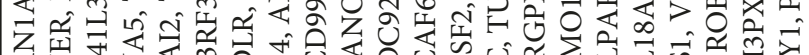

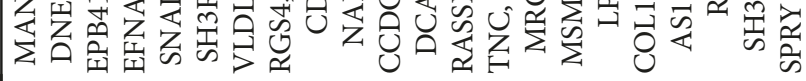

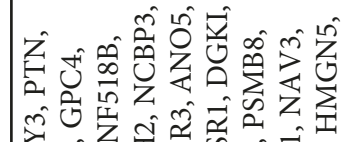

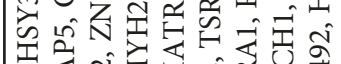

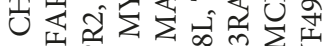

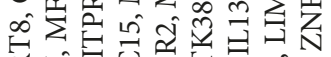

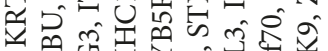

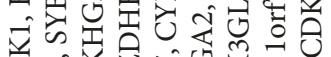

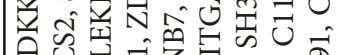
की

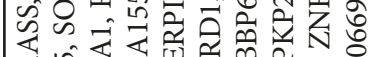

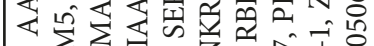
ली

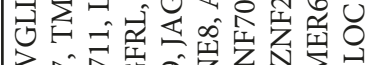

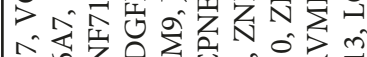

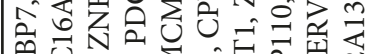

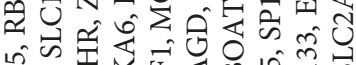

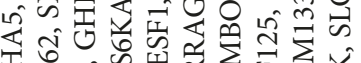

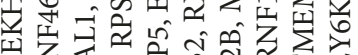
성

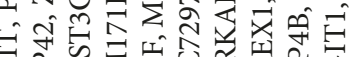

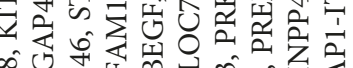

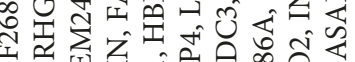

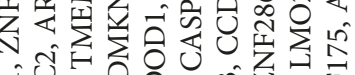
जी

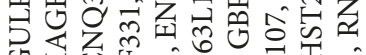

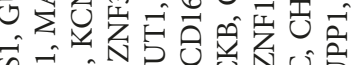
$\vec{p} \vec{\Sigma} \vec{z}=0$

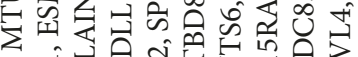

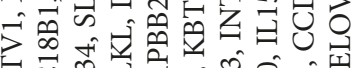

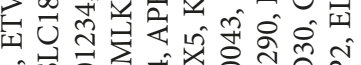
ये हैं

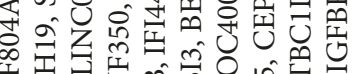

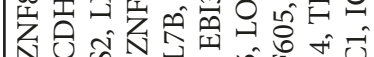

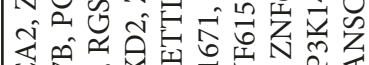

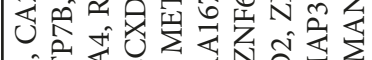

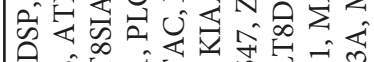

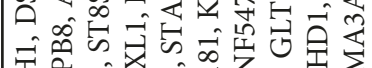

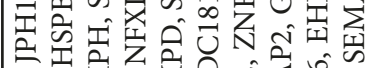

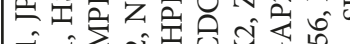

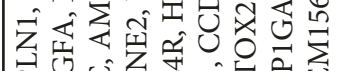

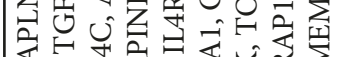

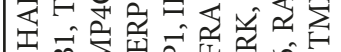

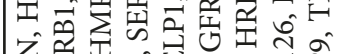

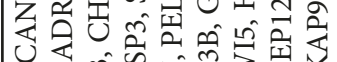

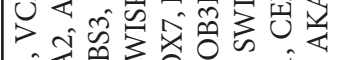
คิ

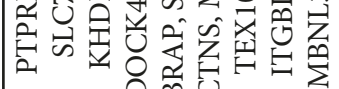

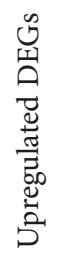

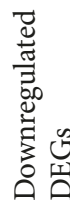




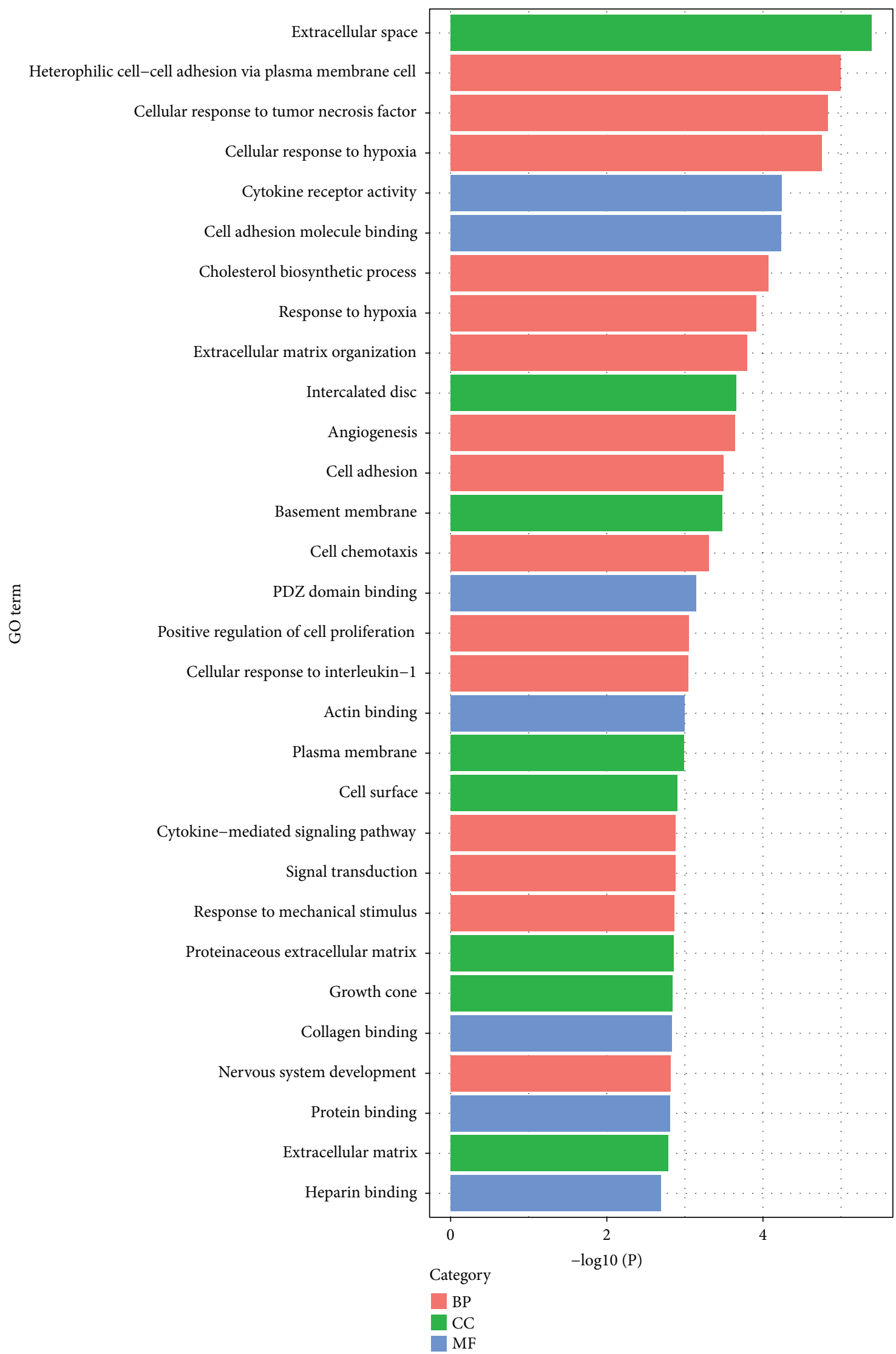

FIGURE 3: Top thirty significant enriched GO terms of DEGs in BC according to their functions. GO: gene ontology; BP: biological process; MF: molecular function; CC: cellular component; DEGs: differentially expressed genes; BC: bladder cancer. 


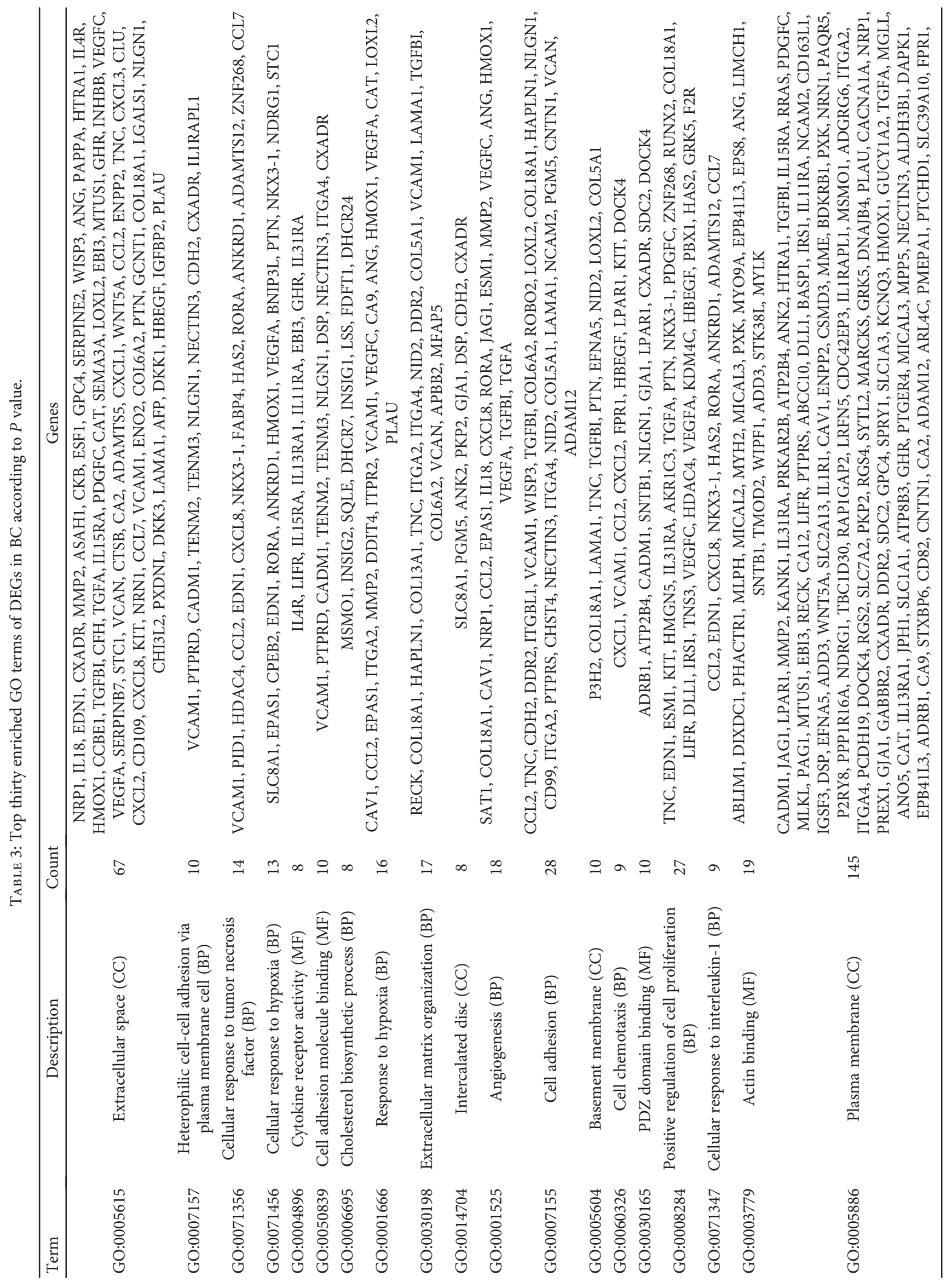




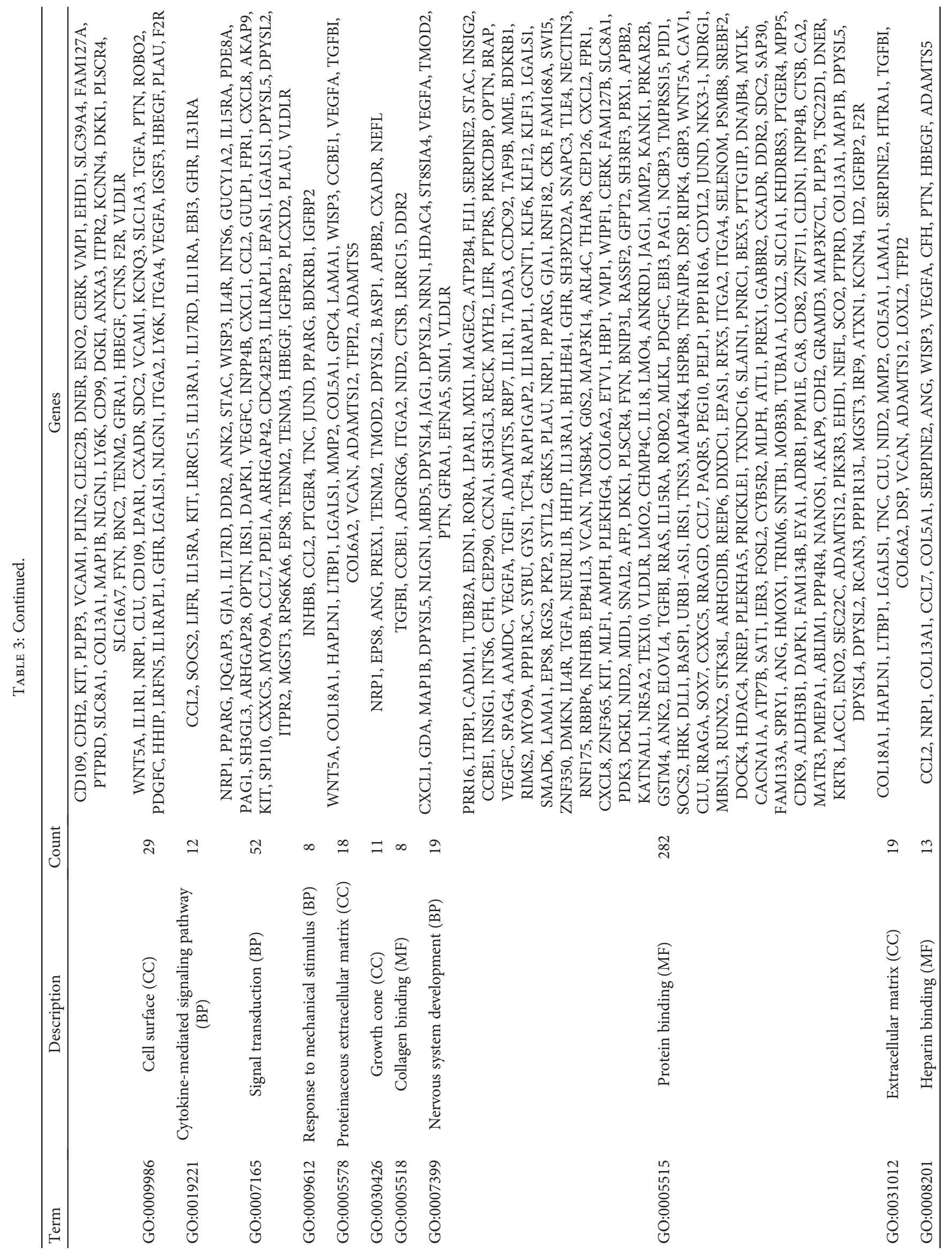




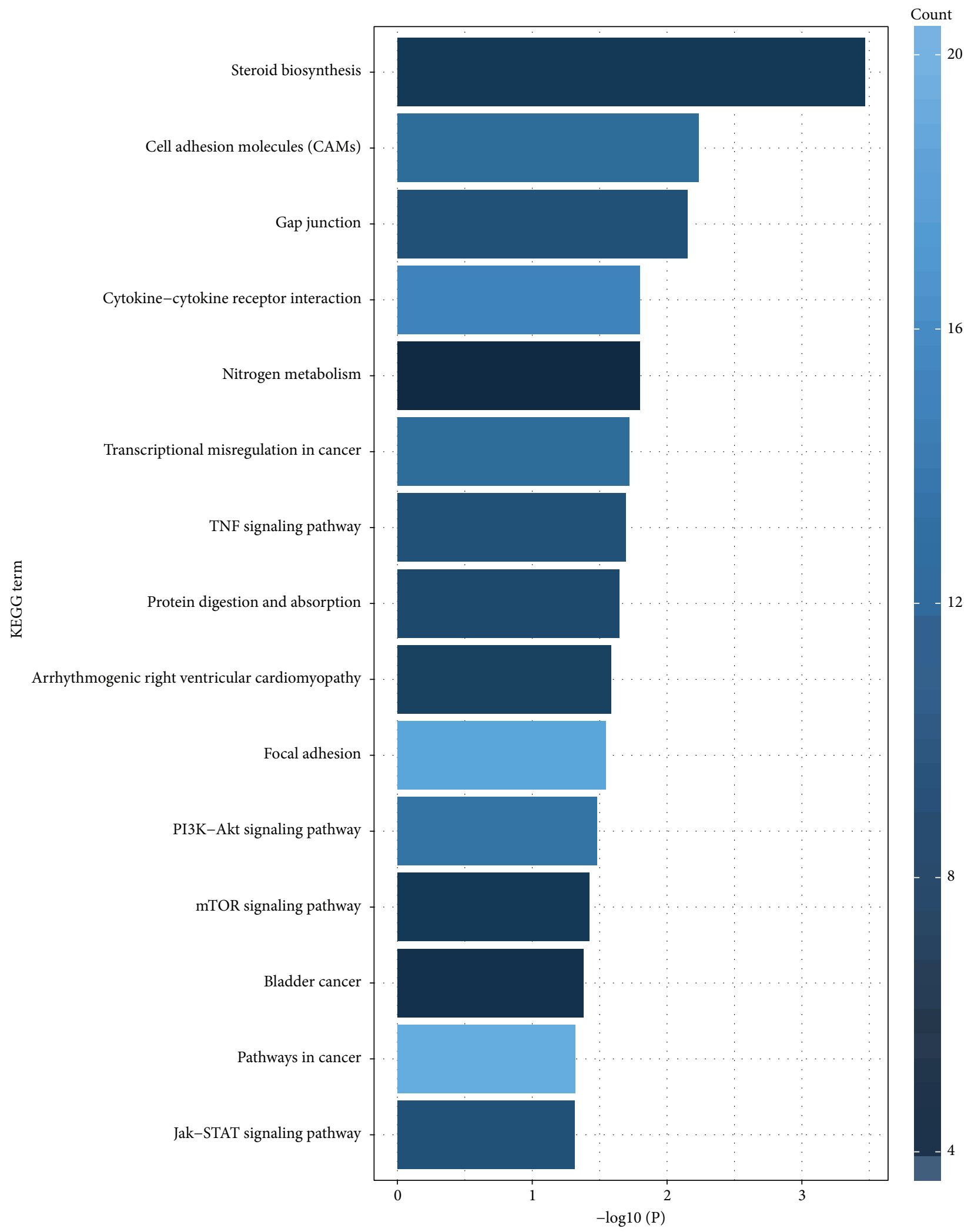

FIGURE 4: Top fifteen significant pathways associated with the DEGs according to $P$ value in KEGG analysis. KEGG: Kyoto Encyclopedia of Genes and Genomes; DEGs: differentially expressed genes.

available literature including in vitro and in vivo studies suggests that melittin governs signal transduction and regulatory pathways associated with multiple cancer death mechanisms, including inhibition of proliferation, induction of apoptosis, inhibition of angiogenesis, cell cycle arrest, and inhibition of cancer motility, migration, metastasis, and invasion [37]. 
TABle 4: Top fifteen significant signaling pathways associated with DEGs in BC according to $P$ value.

\begin{tabular}{|c|c|c|c|c|}
\hline ID & Term & Count & $P$ value & Genes \\
\hline hsa00100 & Steroid biosynthesis & 6 & 0.000342 & MSMO1, SQLE, DHCR7, LSS, FDFT1, DHCR24 \\
\hline hsa04514 & $\begin{array}{l}\text { Cell adhesion molecules } \\
\text { (CAMs) }\end{array}$ & 12 & 0.005828 & $\begin{array}{l}\text { VCAM1, NCAM2, CADM1, CLDN1, NLGN1, CNTN1, CD99, VCAN, } \\
\text { NECTIN3, ITGA4, CDH2, SDC2 }\end{array}$ \\
\hline hsa04540 & Gap junction & 9 & 0.007066 & $\begin{array}{c}\text { ADRB1, TUBB2A, GUCY1A2, TUBAL3, GJA1, PDGFC, LPAR1, TUBA1A, } \\
\text { ITPR2 }\end{array}$ \\
\hline hsa04060 & $\begin{array}{l}\text { Cytokine-cytokine receptor } \\
\text { interaction }\end{array}$ & 15 & 0.015894 & $\begin{array}{l}\text { IL1R1, CCL2, IL18, LIFR, CXCL8, KIT, IL11RA, CCL7, VEGFC, IL4R, VEGFA, } \\
\text { IL15RA, PDGFC, IL13RA1, GHR }\end{array}$ \\
\hline hsa00910 & Nitrogen metabolism & 4 & 0.015944 & CA9, CA8, CA12, CA2 \\
\hline hsa05202 & $\begin{array}{l}\text { Transcriptional } \\
\text { misregulation in cancer }\end{array}$ & 12 & 0.019138 & $\begin{array}{l}\text { EYA1, FLI1, ID2, LMO2, PPARG, CXCL8, CDK9, ETV1, PBX1, RUNX2, } \\
\text { PLAU, MLF1 }\end{array}$ \\
\hline hsa04668 & TNF signaling pathway & 9 & 0.020338 & CXCL1, VCAM1, CCL2, CXCL3, EDN1, CXCL2, MLKL, MAP3K14, PIK3R3 \\
\hline hsa04974 & $\begin{array}{l}\text { Protein digestion and } \\
\text { absorption }\end{array}$ & 8 & 0.022588 & COL18A1, KCNN4, SLC8A1, COL13A1, COL6A2, MME, SLC1A1, COL5A1 \\
\hline hsa05412 & $\begin{array}{l}\text { Arrhythmogenic right } \\
\text { ventricular cardiomyopathy }\end{array}$ & 7 & 0.025957 & PKP2, GJA1, DSP, ITGA2, ITGA4, CDH2, TCF7L1 \\
\hline hsa04151 & PI3K-Akt signaling pathway & 19 & 0.028358 & $\begin{array}{l}\text { TNC, ITGA2, LPAR1, KIT, ITGA4, IRS1, COL5A1, DDIT4, LAMA1, VEGFC, } \\
\text { IL4R, VEGFA, GYS1, COL6A2, EFNA5, PDGFC, PIK3R3, GHR, F2R }\end{array}$ \\
\hline hsa04510 & Focal adhesion & 13 & 0.033173 & $\begin{array}{l}\text { CAV1, TNC, ITGA2, ITGA4, COL5A1, VEGFC, LAMA1, FYN, VEGFA, } \\
\text { COL6A2, PDGFC, PIK3R3, MYLK }\end{array}$ \\
\hline hsa04150 & mTOR signaling pathway & 6 & 0.037778 & RPS6KA6, RRAGA, RRAGD, PIK3R3, IRS1, DDIT4 \\
\hline hsa05219 & Bladder cancer & 5 & 0.041527 & VEGFA, CXCL8, HBEGF, MMP2, DAPK1 \\
\hline hsa05200 & Pathways in cancer & 20 & 0.047909 & $\begin{array}{l}\text { WNT5A, EPAS1, PTGER4, PPARG, CXCL8, ITGA2, BDKRB1, LPAR1, KIT, } \\
\text { MMP2, TCF7L1, DAPK1, LAMA1, VEGFC, VEGFA, TGFA, NKX3-1, HHIP, } \\
\text { PIK3R3, F2R }\end{array}$ \\
\hline hsa04630 & Jak-STAT signaling pathway & 9 & 0.048544 & IRF9, SOCS2, IL4R, LIFR, IL15RA, PIK3R3, IL13RA1, IL11RA, GHR \\
\hline
\end{tabular}

The bold represented the key genes in the modules.

The melittin-related cancers reported consist of leukemia [38], lung carcinoma mouse model [39], pancreatic ductal adenocarcinoma [40], hepatocellular carcinoma cells [41], ovarian cancer cells [11], and so forth. However, little was known about the association between melittin and BC. Melittin and bladder cancer were set as search keywords in the PubMed database, and only three related articles were obtained [42-44]. It was worth noting that the associated signaling pathways involved in melittin regulating $\mathrm{BC}$ also remained yet to be completely elucidated.

In the present work, a genome-wide survey and literature search were therefore conjunctively employed to identify the potential key genes and signaling pathways associated with melittin in BC. 389 upregulated and 169 downregulated DEG genes were obtained according to dataset analysis contingent on high-throughput platforms, enriching the document of BC-related DEGs now available. GO [31] functional enrichment analysis evidenced that most of the DEGs were significantly enriched in protein binding, plasma membrane, extracellular space, and signal transduction. KEGG [32] analysis surfaced that DEGs were essentially abundant in pathways in cancer, PI3K-Akt signal pathway, Jak-STAT signal pathway, TNF signaling pathway, and MTOR signaling pathway. We noted that JAK2/STAT3 [11], TNF- $\alpha$ [15], $\mathrm{mTOR} / \mathrm{p} 70 \mathrm{~S} 6 \mathrm{~K}$ or $\mathrm{mTOR} / \mathrm{p} / 0 \mathrm{~S} 6 \mathrm{~K} / 4 \mathrm{E}-\mathrm{BP} 1$ [16], and PI3K/ Akt/mTOR [17] signaling pathways associated with melittin in cancers were in accordance with the signaling pathway
IDs of hsa04630, hsa04668, hsa04150, and hsa04151. Ulteriorly, fifteen module-related DEGs were obtained according to PPI network and modular analyses with a score no less than 4, namely, CXCL2, CXCL1, BDKRB1, LPAR1, CXCL3, FYN, COL6A2, COL18A1, COL13A1, COL5A1, TUBA1A, FDFT1, SQLE, LSS, and CDK9. Combining KEGG with module analysis, the module-related DEGs corresponding to signaling pathways were described as follows: SQLE, LSS, and FDFT1 for steroid biosynthesis (ID: hsa00100); LPAR1 and TUBA1A for gap junction (ID: hsa04540); CDK9 for transcriptional misregulation in cancer (ID: hsa05202); CXCL1, CXCL3, and CXCL2 for the TNF signaling pathway (ID: hsa04668); COL18A1, COL13A1, COL6A2, and COL5A1 for protein digestion and absorption (ID: hsa04974); LPAR1, COL5A1, and COL6A2 for the PI3K-Akt signaling pathway (ID: hsa04151); COL5A1, FYN, and COL6A2 for focal adhesion (hsa04510); and BDKRB1 and LPAR1 for pathways in cancer (ID: hsa05200). It was clear that the PI3K-Akt (ID: hsa04151) and TNF signaling pathways (ID: hsa04668) associated with melittin contained the module-related DEGs in combination of literature and bioinformatic analyses. The module-related DEGs contained in these two signaling pathways were thus selected for further work. The effect of melittin on their contained module-related DEGs, LPAR1, COL5A1, COL6A2, CXCL1, CXCL2, and CXCL3, was subsequently analyzed in $\mathrm{BC}$ cells. In fact, some of these module-related DEGs have been reported to be significantly correlated with the 


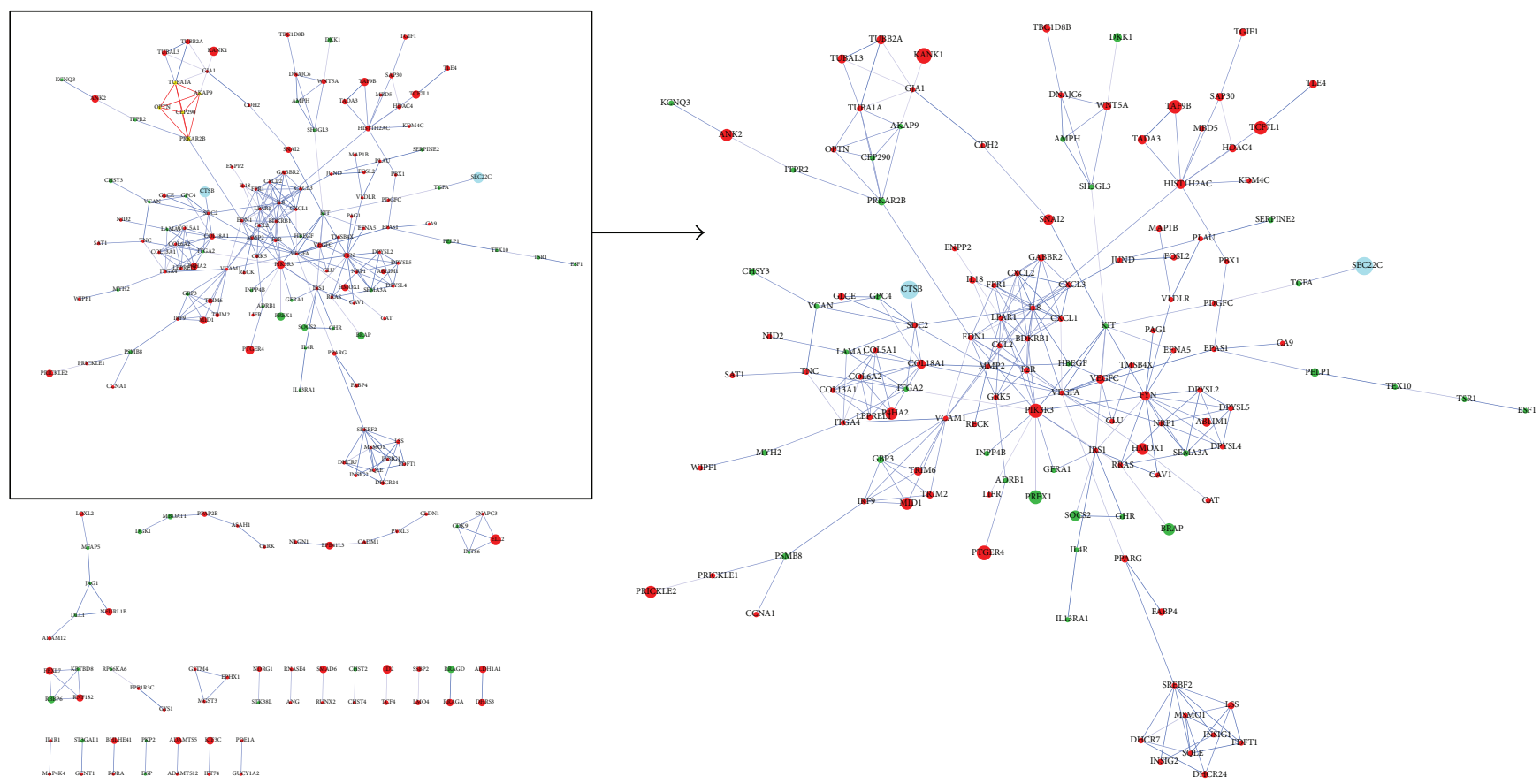

(a)
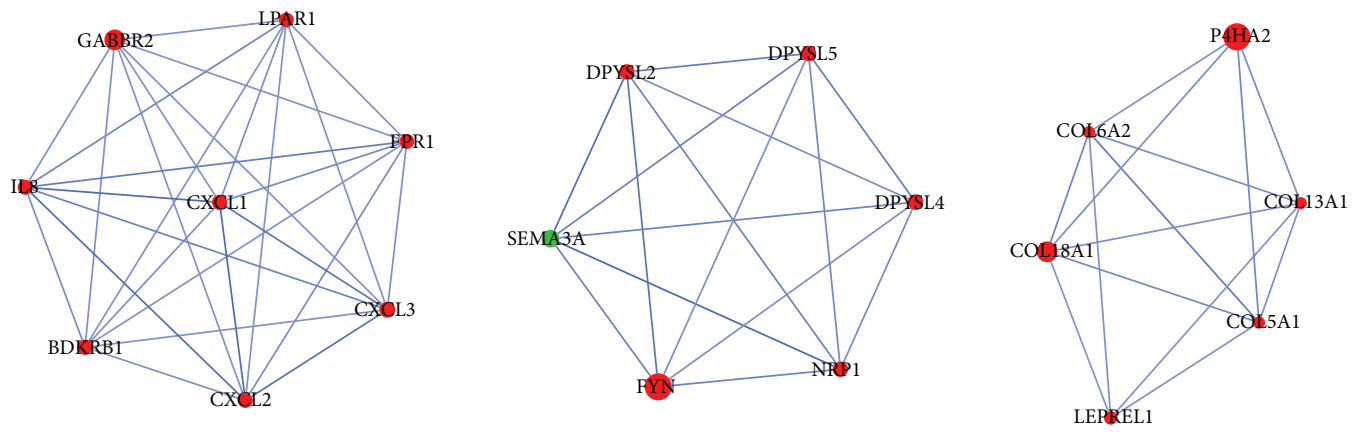

Cluster 1

Cluster 2

Cluster 3

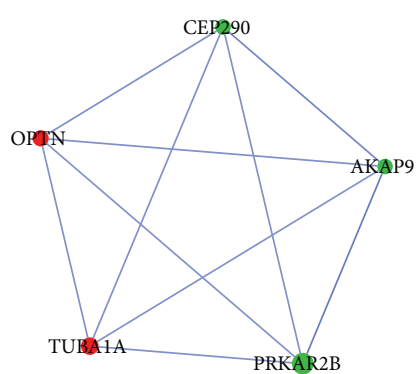

Cluster 4

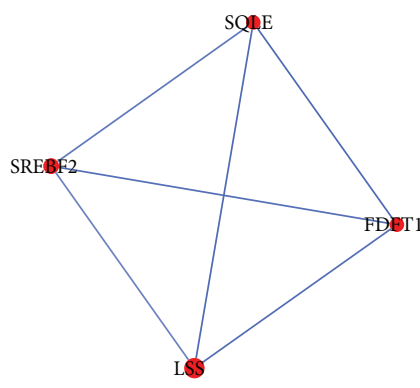

Cluster 5

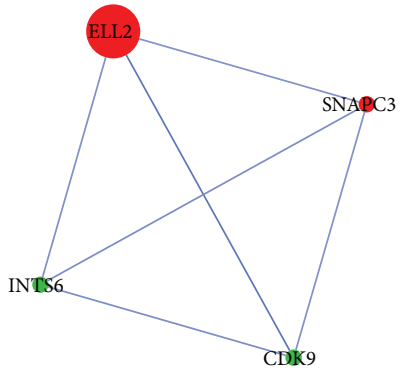

Cluster 6

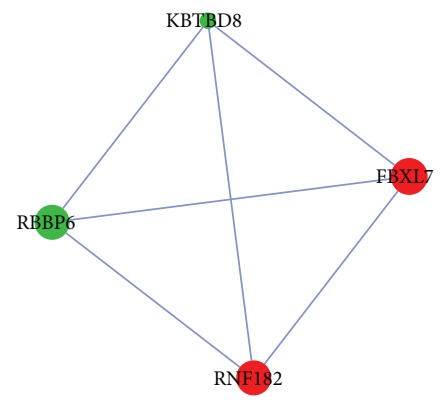

Cluster 7

(b)

FIGURE 5: PPI network constructed by Cytoscape and top seven module networks by MCODE. (a) The PPI network was constructed dependent on the STRING online database (available online: http://string-db.org) and Cytoscape software (version 3.4.0, available online: http://www.cytoscape.org/). (b) The top seven module networks with a score no less than 4 were analyzed by MCODE. The red nodes stand for upregulated genes, and the green nodes stand for downregulated genes. The larger the node diameter is, the smaller the $P$ value and the more significant the node is. PPI: protein-protein interaction; MCODE: Molecular Complex Detection; STRING: Search Tool for the Retrieval of Interacting Genes. 
TABLE 5: Twelve module networks analyzed by MCODE.

\begin{tabular}{lcccc}
\hline Cluster & Score & Nodes & Edges & Node IDs \\
\hline 1 & 8 & 8 & 28 & CXCL2, CXCL1, IL8, GABBR2, FPR1, BDKRB1, LPAR1, CXCL3 \\
2 & 6 & 6 & 15 & SEMA3A, DPYSL4, DPYSL5, DPYSL2, FYN, NRP1 \\
3 & 5.6 & 6 & 14 & COL6A2, P4HA2, COL18A1, COL13A1, COL5A1, LEPREL1 \\
4 & 5 & 5 & 10 & TUBA1A, OPTN, AKAP9, CEP290, PRKAR2B \\
5 & 4 & 4 & 6 & FDFT1, SREBF2, SQLE, LSS \\
6 & 4 & 4 & 6 & SNAPC3, INTS6, CDK9, ELL2 \\
7 & 4 & 6 & 7 & FBXL7, RBBP6, RNF182, KBTBD8 \\
8 & 3.5 & 5 & 3 & TRIM6, IRF9, MID1, TRIM2, GBP3 \\
9 & 3.143 & 3 & 3 & TUBB2A, TUBAL3, GJA1 \\
10 & 3 & 3 & 3 & NEURL1B, DLL1, JAG1 \\
11 & 3 & 3 & & GSTM4, MGST3, EPHX1 \\
12 & 3 & 3 &
\end{tabular}

The bold represented the key genes in the modules.

LPAR1
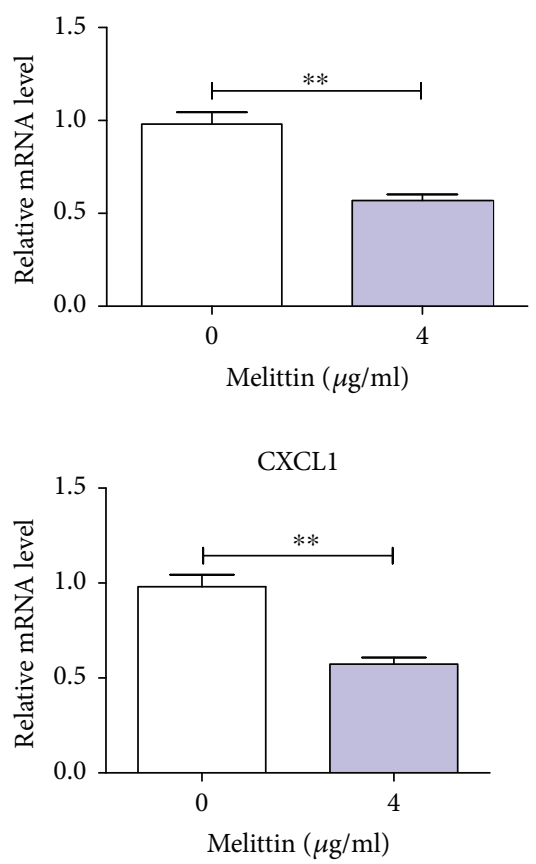

COL5A1

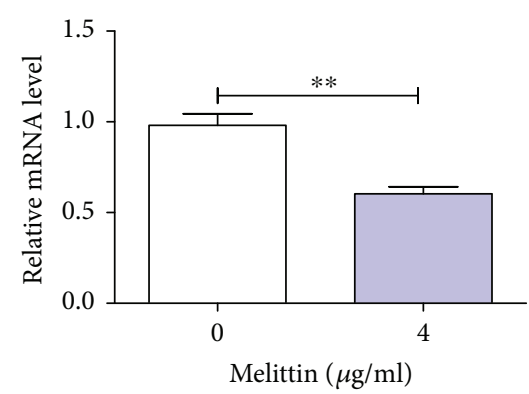

CXCL2

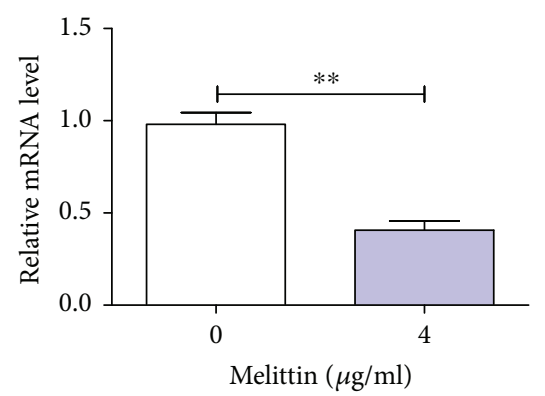

COL6A2

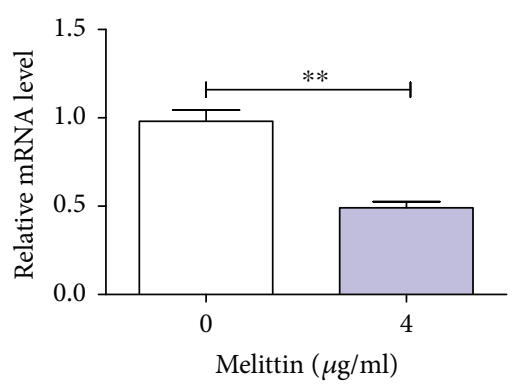

CXCL3

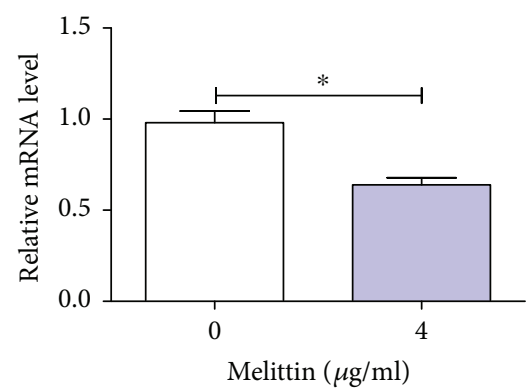

FIGURE 6: The expressions of the module-related DEGs associated with PI3K-Akt and TNF signaling pathways were detected in T24 cells treated with melittin by qRT-PCR. DEGs: differentially expressed genes. ${ }^{*} P<0.05$ and ${ }^{* *} P<0.01$.

occurrence and development of BC, which were exemplified as follows. Zhang et al. reported that the CXCL2-associated signaling pathway, CXCL2/MIF-CXCR2, could advance the recruitment of myeloid-derived suppressor cells and be identified as predictors and potential therapeutic targets in $\mathrm{BC}$ patients [45]. CXCL1 as a potential mediator and marker of the tumor invasion of $\mathrm{BC}$ was elevated in the urine of $\mathrm{BC}$ patients [46, 47]. CXCL1 and CXCL3 related to the nuclear factor kappa B and nuclear factor (erythroid-derived 2)-like 2 signaling pathways were measured in human urothelial carcinoma cell lines [48]. Expression changes of COL3A1 and COL5A1 as the fibrillar collagen proteins were associated with muscle-invasive bladder transition cell carcinoma [49]. Our results showed that these module-related genes were downregulated in cells treated with melittin compared to the control, indicating that melittin could constrain the expression of the module-related DEGs associated with PI3K-Akt and TNF signaling pathways in BC. This further highlights that these genes might be drug-sensitive markers for melittin in BC treatment. As previously reported, melittin exerts an antitumor effect on non-small-cell lung cancer cells [50] and suppresses EGF-induced cell motility and invasion in breast cancer cells [17]. In our work, the functional assays conveyed the prohibitive role of melittin in proliferative and migrated abilities of BC cell lines. This may be the first evidence regarding the initiatory role of melittin in BC metastasis.

To conclude, our findings conveyed valuable information about the mechanism of $\mathrm{BC}$ carcinogenesis and expose the 


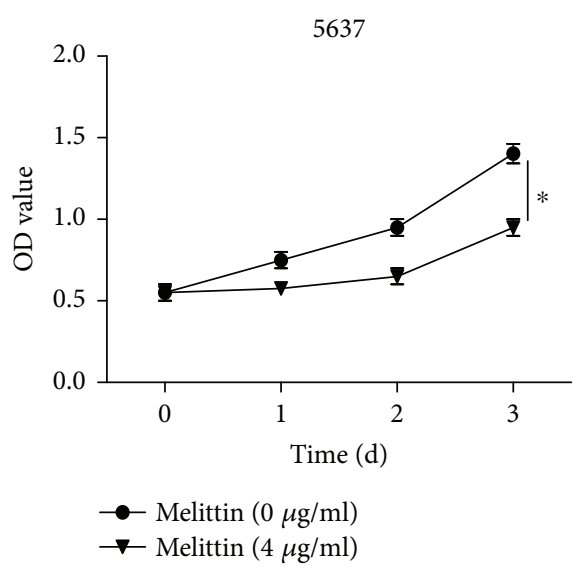

(a)
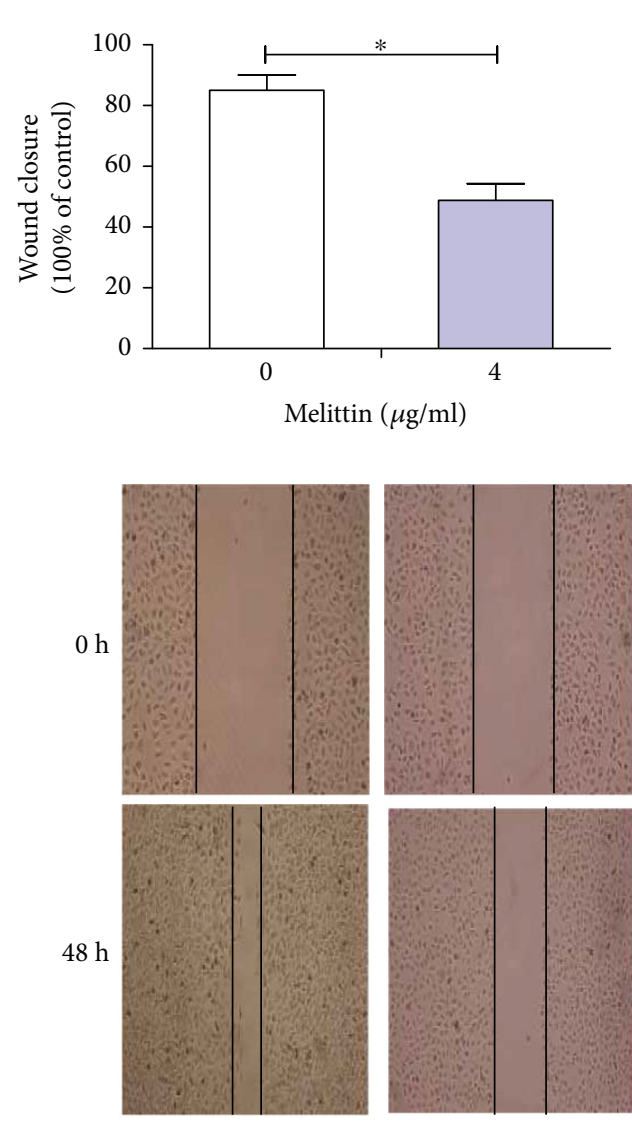

$0 \mu \mathrm{g} / \mathrm{ml}$

(c)
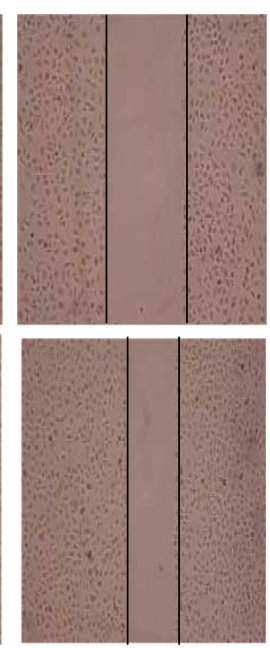

$4 \mu \mathrm{g} / \mathrm{ml}$

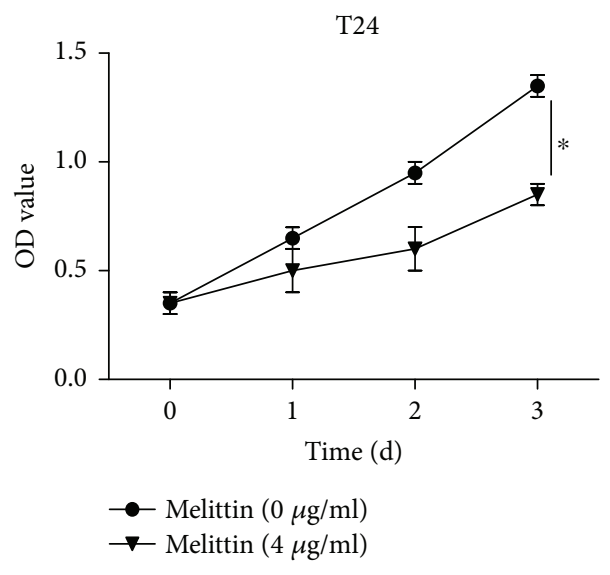

(b)
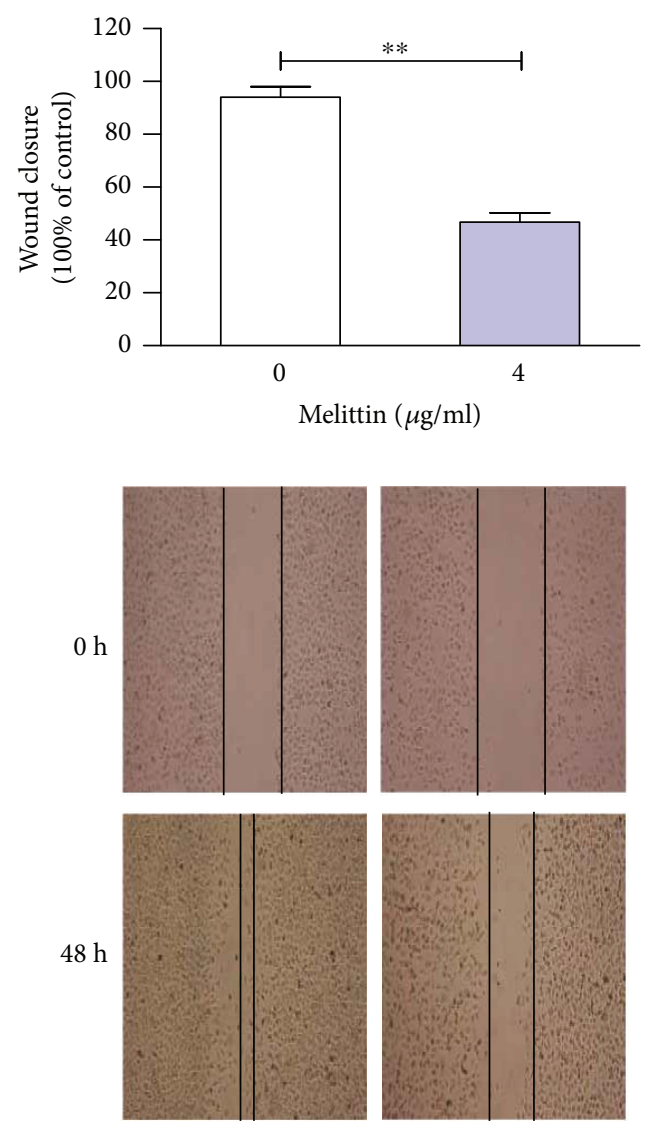

$0 \mu \mathrm{g} / \mathrm{ml}$

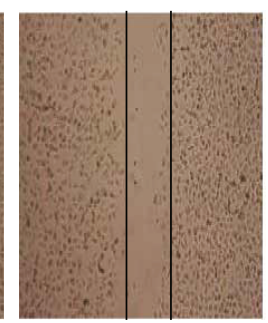

$4 \mu \mathrm{g} / \mathrm{ml}$

(d)

Figure 7: Melittin suppressed cell proliferation and migration in 5637 and T24 cell lines. (a) 5637 and (b) T24 cell viabilities were significantly inhibited in the melittin-treated group ( $4 \mu \mathrm{g} / \mathrm{ml})$ in comparison with the control group $(0 \mu \mathrm{g} / \mathrm{ml})$ as determined by the MTS assay. (c) 5637 and (d) T24 cell migration was remarkably constrained in the melittin-treated group $(4 \mu \mathrm{g} / \mathrm{ml})$ in comparison with the control group $(0 \mu \mathrm{g} / \mathrm{ml})$ as analyzed by the scratch wound-healing assay. Magnification $\times 100$. OD: optical density. ${ }^{*} P<0.05$ and ${ }^{* *} P<0.01$.

discovery of key genes that may act as future targets of anticancer therapy. However, only preliminary verification was conducted regarding the effect of melittin on the expression of the key genes in BC cells in this work, and further investigation remains yet to be elucidated. Therefore, the functional role and concrete mechanism of melittin on the crucial genes in $\mathrm{BC}$ would move forward in our next plan on the basis of this work.

\section{Conclusions}

In this work, we identified 558 candidate DEGs and most of them were significantly enriched in protein binding, plasma membrane, extracellular space, and signal transduction. Fifteen module-related DEGs were obtained, and melittin could constrain the expressions of LPAR1, COL5A1, and COL6A2 
associated with the PI3K-Akt signaling pathway and CXCL1, CXCL2, and CXCL3 involved in the TNF signal pathway in $\mathrm{BC}$ cells. We also found that melittin could induce a significant reduction in proliferation and migration of $\mathrm{BC}$ cells. These findings provide a theoretical basis for these six genes as drug-sensitive markers of melittin in BC treatment.

\section{Abbreviations}

$\begin{array}{ll}\text { BC: } & \text { Bladder cancer } \\ \text { GEO: } & \text { Gene Expression Omnibus } \\ \text { DEGs: } & \text { Differentially expressed genes } \\ \text { GO: } & \text { Gene ontology } \\ \text { KEGG: } & \text { Kyoto Encyclopedia of Genes and Genomes } \\ \text { PPI: } & \text { Protein-protein interaction } \\ \text { DAVID: } & \text { Database for Annotation, Visualization, and } \\ & \text { Integrated Discovery } \\ \text { STRING: } & \text { Search Tool for the Retrieval of Interacting Genes } \\ \text { MCODE: } & \text { Molecular Complex Detection } \\ \text { FBS: } & \text { Fetal bovine serum } \\ \text { BP: } & \text { Biological process } \\ \text { MF: } & \text { Molecular function } \\ \text { CC: } & \text { Cellular component. }\end{array}$

\section{Conflicts of Interest}

The authors declare that there is no conflict of interest regarding the publication of this article.

\section{Authors' Contributions}

Zidan Jin, Jie Yao, Nianlin Xie, and Libo Cai contributed equally to this work.

\section{Acknowledgments}

This work was supported by the National Natural Science Foundation of China (nos. 81673738 and 81703875).

\section{Supplementary Materials}

The PPI network was clearly shown in the Supplementary Material for zooming in and out as needed. The larger the node diameter is, the smaller the $P$ value and the more significant the node is. (Supplementary Materials)

\section{References}

[1] A. F. Ismail, S. Oskay Halacli, N. Babteen et al., "PAK5 mediates cell: cell adhesion integrity via interaction with E-cadherin in bladder cancer cells," Biochemical Journal, vol. 474, no. 8, pp. 1333-1346, 2017.

[2] Z. Kirkali, T. Chan, M. Manoharan et al., "Bladder cancer: epidemiology, staging and grading, and diagnosis," Urology, vol. 66, no. 6, Supplement 1, pp. 4-34, 2005.

[3] X.-R. Wu, "Urothelial tumorigenesis: a tale of divergent pathways," Nature Reviews Cancer, vol. 5, no. 9, pp. 713-725, 2005.

[4] S. Tommasi and A. Besaratinia, "A versatile assay for detection of aberrant DNA methylation in bladder cancer," in Urothelial Carcinoma, W. Schulz, M. Hoffmann, and G. Niegisch, Eds., vol. 1655 of Methods in molecular biology, pp. 29-41, Humana Press, New York, NY, USA, 2017.

[5] O. Rodriguez Faba, J. Palou, H. Vila Reyes et al., "Treatment options and predictive factors for recurrence and cancerspecific mortality in bladder cancer after renal transplantation: a multi-institutional analysis," Actas Urológicas Españolas, vol. 41, no. 10, pp. 639-645, 2017.

[6] W. F. Lin, J. Y. Lu, B. B. Cheng, and C. Q. Ling, "Progress in research on the effects of traditional Chinese medicine on the tumor microenvironment," Journal of Integrative Medicine, vol. 15, no. 4, pp. 282-287, 2017.

[7] Q. Q. Zang, L. Zhang, N. Gao, and C. Huang, "Ophiopogonin $D$ inhibits cell proliferation, causes cell cycle arrest at $G_{2} / M$, and induces apoptosis in human breast carcinoma MCF-7 cells," Journal of Integrative Medicine, vol. 14, no. 1, pp. 51-59, 2016.

[8] B. H. Han, Y. J. Lee, J. J. Yoon et al., "Hwangryunhaedoktang exerts anti-inflammation on LPS-induced NO production by suppressing MAPK and NF- $\kappa$ B activation in RAW264.7 macrophages," Journal of Integrative Medicine, vol. 15, no. 4, pp. 326-336, 2017.

[9] D. Eisenberg, "Three-dimensional structure of membrane and surface proteins," Annual Review of Biochemistry, vol. 53, no. 1, pp. 595-623, 1984.

[10] R. C. Hider, "Honeybee venom: a rich source of pharmacologically active peptides," Endeavour, vol. 12, no. 2, pp. 60-65, 1988.

[11] M. Jo, M. Park, P. Kollipara et al., "Anti-cancer effect of bee venom toxin and melittin in ovarian cancer cells through induction of death receptors and inhibition of JAK2/STAT3 pathway," Toxicology and Applied Pharmacology, vol. 258, no. 1, pp. 72-81, 2012.

[12] M. H. Park, M. S. Choi, D. H. Kwak et al., "Anti-cancer effect of bee venom in prostate cancer cells through activation of caspase pathway via inactivation of NF- $\kappa \mathrm{B}$," The Prostate, vol. 71, no. 8, pp. 801-812, 2011.

[13] J. H. Park, Y. J. Jeong, K. K. Park et al., "Melittin suppresses PMA-induced tumor cell invasion by inhibiting NF- $\kappa \mathrm{B}$ and AP-1-dependent MMP-9 expression," Molecules and Cells, vol. 29, no. 2, pp. 209-215, 2010.

[14] H. J. Cho, Y. J. Jeong, K. K. Park et al., "Bee venom suppresses PMA-mediated MMP-9 gene activation via JNK/p38 and NF$\kappa \mathrm{B}$-dependent mechanisms," Journal of Ethnopharmacology, vol. 127, no. 3, pp. 662-668, 2010.

[15] Y. J. Jeong, H. J. Cho, K. Whang et al., "Melittin has an inhibitory effect on TNF- $\alpha$-induced migration of human aortic smooth muscle cells by blocking the MMP-9 expression," Food and Chemical Toxicology, vol. 50, no. 11, pp. 3996-4002, 2012.

[16] J. Shin, Y. Jeong, H. Cho et al., "Melittin suppresses HIF-1 $\alpha /$ VEGF expression through inhibition of ERK and mTOR/ p70S6K pathway in human cervical carcinoma cells," PLoS One, vol. 8, no. 7, article e69380, 2013.

[17] Y. Jeong, Y. Choi, J. Shin et al., "Melittin suppresses EGFinduced cell motility and invasion by inhibiting PI3K/Akt/ mTOR signaling pathway in breast cancer cells," Food and Chemical Toxicology, vol. 68, pp. 218-225, 2014.

[18] G. M. Kong, W. H. Tao, Y. L. Diao et al., "Melittin induces human gastric cancer cell apoptosis via activation of mitochondrial pathway," World Journal of Gastroenterology, vol. 22, no. 11, pp. 3186-3195, 2016. 
[19] J. E. Huh, J. W. Kang, D. Nam et al., "Melittin suppresses VEGF-A-induced tumor growth by blocking VEGFR-2 and the COX-2-mediated MAPK signaling pathway," Journal of Natural Products, vol. 75, no. 11, pp. 1922-1929, 2012.

[20] J. H. Park, B. Park, and K. K. Park, "Suppression of hepatic epithelial-to-mesenchymal transition by melittin via blocking of TGF $\beta /$ Smad and MAPK-JNK signaling pathways," Toxins, vol. 9, no. 4, p. 138, 2017.

[21] G. Qin, Y. Chen, H. Li et al., "Melittin inhibits tumor angiogenesis modulated by endothelial progenitor cells associated with the SDF- $1 \alpha /$ CXCR4 signaling pathway in a UMR-106 osteosarcoma xenograft mouse model," Molecular Medicine Reports, vol. 14, no. 1, pp. 57-68, 2016.

[22] V. Kulasingam and E. Diamandis, "Strategies for discovering novel cancer biomarkers through utilization of emerging technologies," Nature Clinical Practice Oncology, vol. 5, no. 10, pp. 588-599, 2008.

[23] Z. Zhang, G. Zhang, Z. Gao et al., "Comprehensive analysis of differentially expressed genes associated with PLK1 in bladder cancer," BMC Cancer, vol. 17, no. 1, p. 861, 2017.

[24] X. Ning and Y. Deng, "Identification of key pathways and genes influencing prognosis in bladder urothelial carcinoma," OncoTargets and Therapy, vol. 10, pp. 1673-1686, 2017.

[25] D. Q. Zhang, C. K. Zhou, S. Z. Chen, Y. Yang, and B. K. Shi, "Identification of hub genes and pathways associated with bladder cancer based on co-expression network analysis," Oncology Letters, vol. 14, no. 1, pp. 1115-1122, 2017.

[26] D. B. Allison, X. Cui, G. P. Page, and M. Sabripour, "Microarray data analysis: from disarray to consolidation and consensus," Nature Reviews Genetics, vol. 7, no. 1, pp. 55-65, 2006.

[27] L. Mengual, M. Burset, E. Ars et al., "DNA microarray expression profiling of bladder cancer allows identification of noninvasive diagnostic markers," The Journal of Urology, vol. 182, no. 2, pp. 741-748, 2009.

[28] Y. D. Huang, W. Shan, L. Zeng, and Y. Wu, "Screening of differentially expressed genes related to bladder cancer and functional analysis with DNA microarray," Asian Pacific Journal of Cancer Prevention, vol. 14, no. 8, pp. 4553-4557, 2013.

[29] D. Theodorescu, L. M. Sapinoso, M. R. Conaway, G. Oxford, G. M. Hampton, and Frierson HF Jr, "Reduced expression of metastasis suppressor RhoGDI2 is associated with decreased survival for patients with bladder cancer," Clinical Cancer Research, vol. 10, no. 11, pp. 3800-3806, 2004.

[30] M. E. Ritchie, B. Phipson, D. Wu et al., "Limma powers differential expression analyses for RNA-sequencing and microarray studies," Nucleic Acids Research, vol. 43, no. 7, article e47, 2015.

[31] M. Ashburner, C. A. Ball, J. A. Blake et al., "Gene ontology: tool for the unification of biology," Nature Genetics, vol. 25, no. 1, pp. 25-29, 2000.

[32] J. Du, Z. Yuan, Z. Ma, J. Song, X. Xie, and Y. Chen, "KEGGPATH: Kyoto encyclopedia of genes and genomes-based pathway analysis using a path analysis model," Molecular BioSystems, vol. 10, no. 9, pp. 2441-2447, 2014.

[33] D. S. Murrell, I. Cortes-Ciriano, G. J. P. van Westen et al., "Chemically aware model builder (camb): an R package for property and bioactivity modelling of small molecules," Journal of Cheminformatics, vol. 7, no. 1, p. 45, 2015.

[34] A. Franceschini, D. Szklarczyk, S. Frankild et al., "STRING v9.1: protein-protein interaction networks, with increased coverage and integration," Nucleic Acids Research, vol. 41, no. D1, pp. D808-D815, 2013.
[35] G. Su, J. H. Morris, B. Demchak, and G. D. Bader, "Biological network exploration with Cytoscape 3," Current Protocols in Bioinformatics, vol. 47, pp. 8.13.1-8.13.24, 2014.

[36] G. D. Bader and C. W. Hogue, "An automated method for finding molecular complexes in large protein interaction networks," BMC Bioinformatics, vol. 4, no. 1, p. 2, 2003.

[37] I. Rady, I. A. Siddiqui, M. Rady, and H. Mukhtar, "Melittin, a major peptide component of bee venom, and its conjugates in cancer therapy," Cancer Letters, vol. 402, pp. 16-31, 2017.

[38] D. O. Moon, S. Y. Park, Y. H. Choi, N. D. Kim, C. Lee, and G. Y. Kim, "Melittin induces Bcl-2 and caspase-3-dependent apoptosis through downregulation of Akt phosphorylation in human leukemic U937 cells," Toxicon, vol. 51, no. 1, pp. 112-120, 2008.

[39] C. Lee, S. S. Bae, H. Joo, and H. Bae, "Melittin suppresses tumor progression by regulating tumor-associated macrophages in a Lewis lung carcinoma mouse model," Oncotarget, vol. 8, no. 33, pp. 54951-54965, 2017.

[40] X. Wang, J. Xie, X. Lu et al., "Melittin inhibits tumor growth and decreases resistance to gemcitabine by downregulating cholesterol pathway gene CLU in pancreatic ductal adenocarcinoma," Cancer Letters, vol. 399, pp. 1-9, 2017.

[41] X. Wu, B. Zhao, Y. Cheng et al., "Melittin induces PTCH1 expression by down-regulating MeCP2 in human hepatocellular carcinoma SMMC-7721 cells," Toxicology and Applied Pharmacology, vol. 288, no. 1, pp. 74-83, 2015.

[42] N. Orsolic, "Bee venom in cancer therapy," Cancer Metastasis Reviews, vol. 31, no. 1-2, pp. 173-194, 2012.

[43] T. Carter, K. Sterling-Levis, K. Ow et al., "Biodistributions of intact monoclonal antibodies and fragments of BLCA-38, a new prostate cancer directed antibody," Cancer Immunology, Immunotherapy, vol. 53, no. 6, pp. 533-542, 2004.

[44] D. Winder, W. H. Günzburg, V. Erfle, and B. Salmons, "Expression of antimicrobial peptides has an antitumour effect in human cells," Biochemical and Biophysical Research Communications, vol. 242, no. 3, pp. 608-612, 1998.

[45] H. Zhang, Y. L. Ye, M. X. Li et al., "CXCL2/MIF-CXCR2 signaling promotes the recruitment of myeloid-derived suppressor cells and is correlated with prognosis in bladder cancer," Oncogene, vol. 36, no. 15, pp. 2095-2104, 2017.

[46] A. Burnier, Y. Shimizu, Y. Dai et al., "CXCL1 is elevated in the urine of bladder cancer patients," SpringPlus, vol. 4, no. 1, p. 610, 2015.

[47] H. Kawanishi, Y. Matsui, M. Ito et al., "Secreted CXCL1 is a potential mediator and marker of the tumor invasion of bladder cancer," Clinical Cancer Research, vol. 14, no. 9, pp. 25792587, 2008.

[48] G. Shah, G. Zhang, F. Chen, Y. L. Cao, B. Kalyanaraman, and W. A. See, "iNOS expression and NO production contribute to the direct effects of BCG on urothelial carcinoma cell biology," Urologic Oncology: Seminars and Original Investigations, vol. 32, no. 1, pp. 45.e1-45.e9, 2014.

[49] J. A. Ewald, T. M. Downs, J. P. Cetnar, and W. A. Ricke, "Expression microarray meta-analysis identifies genes associated with Ras/MAPK and related pathways in progression of muscle-invasive bladder transition cell carcinoma," PLoS One, vol. 8, no. 2, article e55414, 2013.

[50] S. F. Zhang and Z. Chen, "Melittin exerts an antitumor effect on non-small cell lung cancer cells," Molecular Medicine Reports, vol. 16, no. 3, pp. 3581-3586, 2017. 


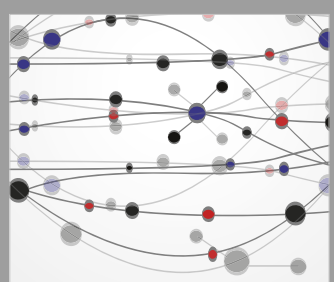

The Scientific World Journal
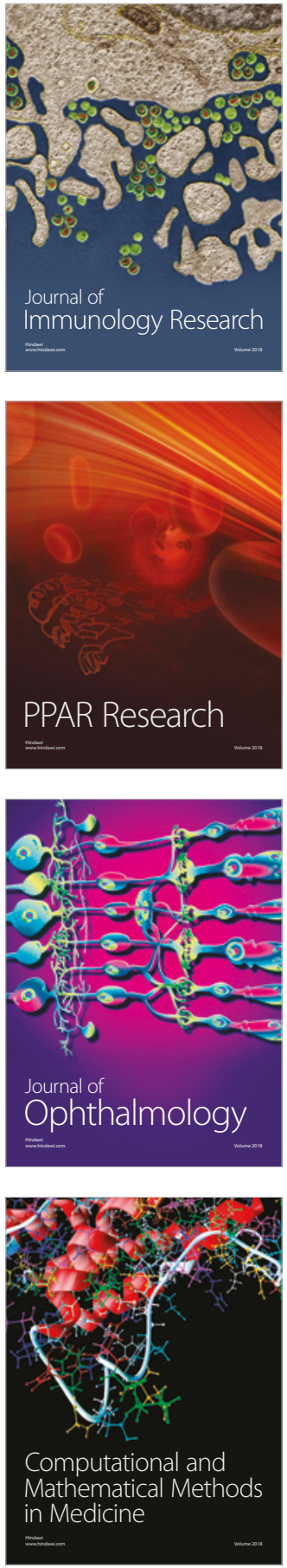

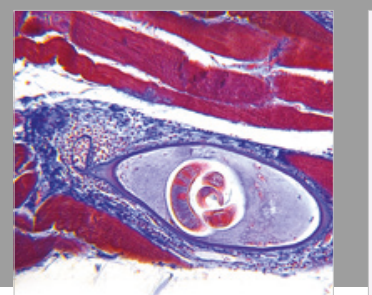

Gastroenterology Research and Practice

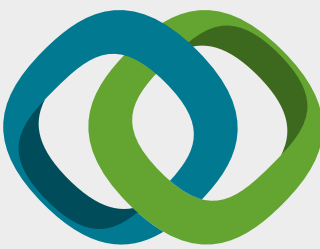

\section{Hindawi}

Submit your manuscripts at

www.hindawi.com
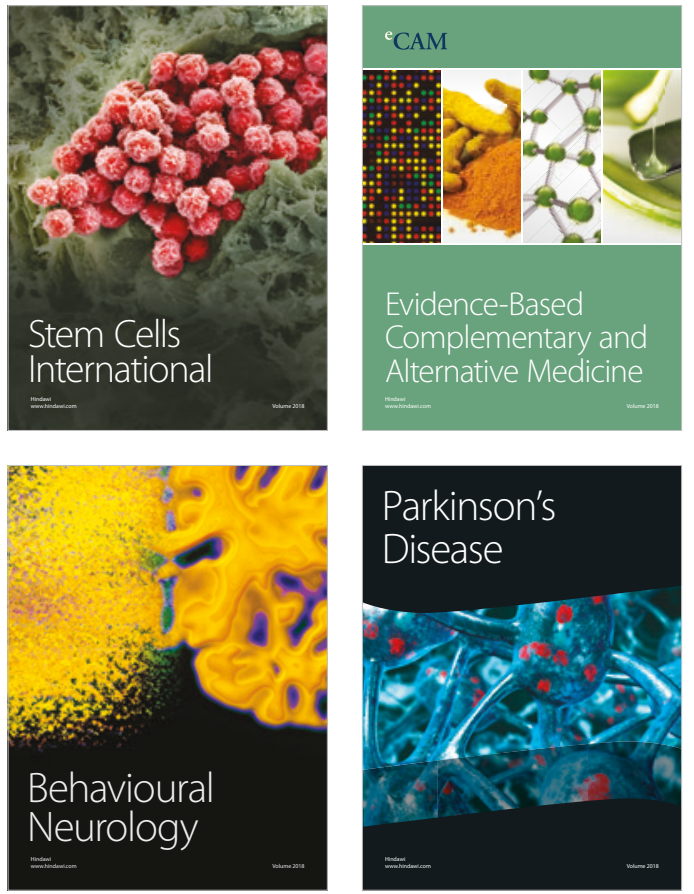

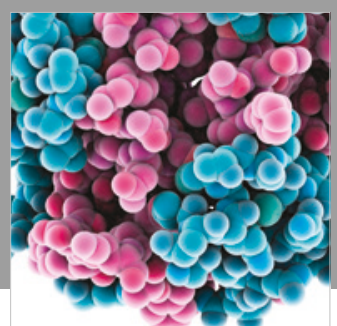

ournal of

Diabetes Research

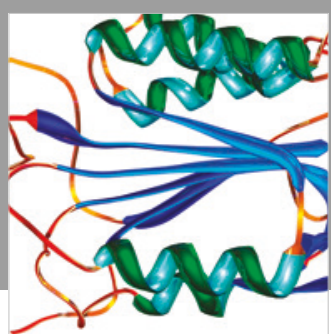

Disease Markers
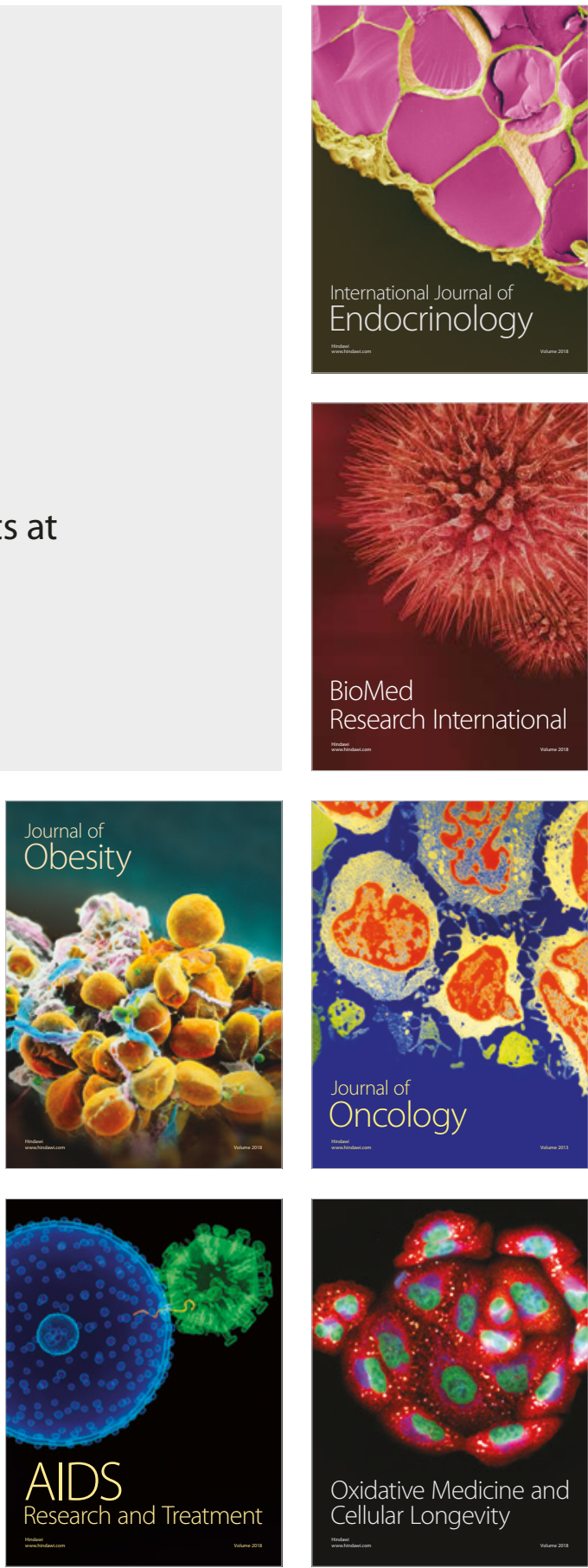